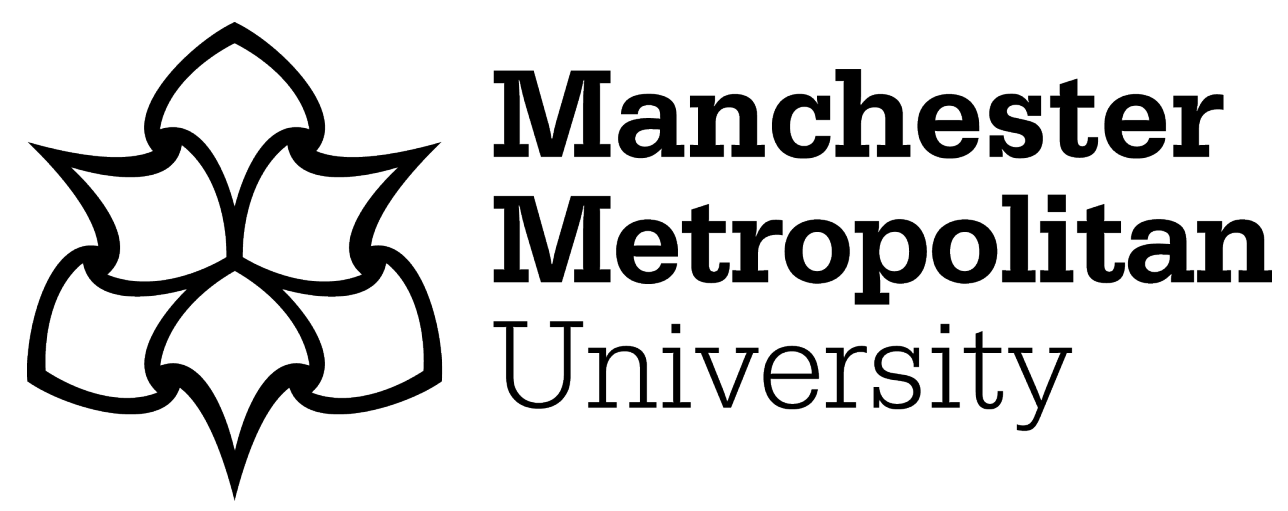

Chen, H, Qian, L ORCID logoORCID: https://orcid.org/0000-0002-97162342, Bai, W ORCID logoORCID: https://orcid.org/0000-0002-3537-207X, $\mathrm{Ma}, \mathrm{Z}$ ORCID logoORCID: https://orcid.org/0000-0002-2426-3038, Lin, Z and Xue, MA (2019) Oblique focused wave group generation and interaction with a fixed FPSO-shaped body: 3D CFD simulations and comparison with experiments. Ocean Engineering, 192. p. 106524. ISSN 0029-8018

Downloaded from: https://e-space.mmu.ac.uk/624263/

Version: Accepted Version

Publisher: Elsevier

DOI: https://doi.org/10.1016/j.oceaneng.2019.106524

Usage rights: Creative Commons: Attribution-Noncommercial-No Derivative Works 4.0

Please cite the published version 


\title{
Oblique focused wave group generation and interaction with a fixed FPSO-shaped body: 3D CFD simulations and comparison with experiments
}

\author{
Hao Chen ${ }^{\mathrm{a}, *}$, Ling Qian ${ }^{\mathrm{a}, * *}$, Wei Bai ${ }^{\mathrm{a}}$, Zhihua Ma $^{\mathrm{a}}$, Zaibin Lin $^{\mathrm{a}}$, Mi-An Xue ${ }^{\mathrm{b}}$ \\ ${ }^{a}$ Centre for Mathematical Modelling and Flow Analysis, School of Computing, Mathematics and Digital Technology, \\ Manchester Metropolitan University, Manchester, United Kingdom \\ ${ }^{b}$ College of Harbour Coastal and Offshore Engineering, Hohai University, Nanjing 210098, China
}

\begin{abstract}
This paper presents a numerical study of oblique focused wave group generation and interaction with a fixed FPSO-shaped body, with thorough validations against available experimental data. The 3D numerical model is based on the open-source toolbox OpenFOAM ${ }^{\circledR}$, where the oblique waves are generated using multiple virtual segmented wave paddles. The surface elevation and velocity profiles on each paddle are derived based on the snake principle, which mimics the behaviour of wave paddles in the physical wave tank. Numerical tests are firstly conducted for focused wave groups propagating obliquely in an empty wave tank using the proposed scheme. By analysis of the surface elevation, it is found that reasonably good quality of oblique wave fields can be generated in the central area of the wave basin. Furthermore, investigations are carried out on the effects of wave angles on the harmonic structures of the wave groups using the phase-inversion method. It is shown that while the wave angle has minor effects on the linear and second order harmonics, the third order harmonic is altered by the wave angles, albeit its magnitude is very small. Finally, to show the effectiveness of the numerical oblique wave generation method and the importance of the angle effects in the wave-structure interaction process, simulations are carried out for the oblique focused wave group interacting with a fixed FPSO-shaped body. The effects of the wave incidence angle are clearly shown from the comparison of the integrated wave forces between the cases with different wave propagating angles.
\end{abstract}

Keywords:

Oblique waves; Focused wave groups; Wave-maker theory.

\section{Introduction}

The floating production storage and offloading (FPSO) vessels are commonly used in offshore oil and gas industry for production and processing of hydrocarbons, and for the storage of oil. With the expansion of oil and gas industry, they are being designed to work in increasingly deep water of potentially harsh marine environment, e.g. during storms and hurricanes. In contrast with ships or naval vessels, FPSOs are usually moored in a specific location for a relatively long time. Therefore, it is of vital importance to examine the survivability of FPSO in such extreme sea states.

A number of physical mechanisms have been proposed regarding the formation of extreme waves, which include spatial focusing, dispersive focusing and nonlinear focusing (Dysthe et al. 2008). Spatial focusing can be achieved by wave refraction due to varying seabed topologies. Along irregular coastlines, this may lead to the focusing of wave energy in particular places, which provide suitable locations for wave power devices Halliday and Dorrell (2004). However, this mainly occurs in shallow or intermediate water area where

\footnotetext{
${ }^{*}$ Current address: Department of Civil and Environmental Engineering, National University of Singapore, Singapore. e-mail address: ceeche@nus.edu.sg.

${ }^{* *}$ Corresponding author, tel.: +44 (0)161 247 1548; e-mail address: l.qian@mmu.ac.uk.
} 
seabed bottom plays a critical role on the wave propagation. Meanwhile, modulation instability, based on the breather solutions to the nonlinear Schrodinger equation is also an important reason for the extreme waves, see e.g. Peregrine (1983); Chabchoub et al. (2011); Karjanto and Van Groesen (2010). However, in the present work, we only focus on the extreme waves due to dispersive focusing.

The dispersive focused wave groups have been used to represent the statistically expected extreme wave profile based on the NewWave theory (Tromans et al., 1991). The NewWave theory assumes that the profile of extreme waves in a random Gaussian sea can be represented by a suitably scaled focused wave group with its elevation proportional to the auto-correlation function of the underlying random process (Vyzikas et al. 2018). A recent field study also related the occurrence of extreme events to the dispersive focusing of the most energetic wave components (Christou and Ewans, 2014), so that the NewWave-type waves were actually formed. Comparing to application of the regular waves with the corresponding wave height and period, it is believed that the use of focused waves can generally better represent the spectral broadband properties of ocean waves (Chen et al., 2018).

However, ocean waves are multi-directional irregular waves, of which the energy is distributed over a wide range of both frequencies and directions. As well reported in the literature (Ji et al. (2015a b); Li et al. (2012, 2014b) ), the directionality of the wave field plays a critical role in determining the wave kinematics, and essentially the wave loads on offshore structure. Ji et al. (2015b) computed the wave run-up and wave forces on a single cylinder under multi-directional irregular wave conditions, where they found that the front run-up increases but the rear run-up decreases, as the directional distribution becomes narrower. Ji et al. (2015a) further extended their model to an array of cylinders, and they concluded that the combination of wave spreading parameter and the arrangement of the array of cylinders determines the wave run-up and wave forces on the cylinders. Li et al. (2000) applied a Boussinesq-type solver to simulate multi-directional irregular wave diffraction around a semi-infinite breakwater. It was shown that the directional spreading of the incident waves has a significant effect on the wave diffraction, leading to a distinct diffraction contour compared to the case under uni-directional waves. Hereby when evaluating the extreme wave load on FPSOs, of which the geometry is non-axisymmetric, directionality effects should be taken into account. The cases of incident focused wave groups propagating in different angles should be considered.

In many physical wave tank tests, relatively wide rectangular basins have been used, in which directional seas can be generated by prescribing the motion of an array of segmented wave paddles located along one or more sides of the basin based on the so-called "snake-principle" (Dean and Dalrymple, 1991). This theory is derived by assuming that the width of wave paddle tends to infinitely small. Therefore, the displacement of the segmented wave paddles are continuous. Maenwhile, the width of the wave-maker side is assumed to be infinitely long. However, physical wave basins have finite dimensions, which changes wave generation theory in a fundamental manner, as waves are reflected from the basin walls, and there is no energy radiation (Newman, 2010). Therefore, the segmented wave-makers can only reproduce the directional waves within a limited area, inside which the generated waves can be used for the model test. In Li et al. (2014a), the size and shape of this effective testing area were investigated experimentally under short-crested waves.

With regard to the numerical simulations, different methods have been proposed for wave generations. For potential flow solver, Ducrozet et al. (2006) applied a high order spectral methods, where the wave-maker boundary condition was expressed as a no-flux boundary condition plus an additional potential specially for wave-making. In Williams and Crull (2000), the governing equations for the potential flow were solved by an integral equation technique. The unknown potential on the wave-maker boundary was determined by the snake-principle. Chen (2017) extended the functionality of the numerical wave tank developed in Bai and Eatock Taylor (2006, 2007) using high order boundary element methods, which is shown to be capable of generating oblique regular waves and 3D focusing wave groups.

When it comes to the numerical wave tanks based on the solution of the Navier-Stokes equations, mainly three methodologies are being used for wave generations, namely by adding internal sources, directly modelling wave-maker motions, and impose of Dirichlet boundary conditions based on the wave properties. Internal wave-makers have been used in Ha et al. (2013), where a momentum source term was added in order to drive various kinds of waves. Direct modelling of wave-maker motion has been seen in e.g. Dao et al. (2018); Martínez-Ferrer et al. (2018); Higuera et al. (2015). Actually, better agreement with experiments could be expected when using this method, as the wave-maker motion is explicitly modelled. However, 
the downside is the computational cost, as extra effort is taken by solving the mesh motion equations. Furthermore, one also needs to deal with the discontinuity of motion between paddles, when directional waves are generated. Certain smooth functions need to be introduced to make the mesh motion continuous at the wave-maker boundary. Meanwhile, Direct impose of Dirichlet type boundary conditions is still one of the most widely used wave generation methods, which has been used to generate normal uni-directional focused wave groups as shown in Cao and Wan $(2014) ; \mathrm{Hu}$ et al. (2016); Kim et al. (2001); Ojieh et al. (2009).

In the present work, we will implement a set of boundary conditions to generate oblique uni-directional focused wave groups under different propagation angles in a large 3D numerical wave basin. The modelling framework is based on the open-source toolbox OpenFOAM ${ }^{\circledR}$. The boundary conditions mimic the way oblique waves are generated in laboratory tests by introducing certain amount of flux on each virtual wave paddle. By doing this, it is expected that closer agreement between experimental and simulation results are obtained. The quality of the generated wave groups are assessed, where the surface elevation is compared to the available experimental data. Furthermore, harmonic analysis is carried out and the effect of wave angle on the harmonic structure of the generated focused wave groups is thoroughly analysed. Finally, the angle effects of the extreme wave loads on the FPSO-shape body are evaluated, where the body is subjected to the focused wave groups with three different incident wave angles.

\section{Generation of oblique focused wave groups}

\subsection{Overview}

The present numerical model applies the wave generation toolbox olaFlow, which was based on and further developed from IHFOAM (Higuera et al., 2013), to generate regular and irregular waves. By using this toolbox, waves are produced at a fixed boundary by means of special Dirichlet-type boundary conditions.

For a two-phase Navier-Stokes solver where the interface is tracked by volume of fluid method (see Section 3.2 for details of the model), the typical unknowns are the volume fraction field $\alpha$, the velocity field $\boldsymbol{u}$ and the dynamic pressure $p^{*}$. They need to be properly defined on the wave-maker boundary. Actually, the surface elevation and the velocity are directly extracted from the underlying wave theory. Meanwhile, the pressure is obtained as a part of the solution from PISO algorithm. Therefore, assignment of the boundary condition for the velocity and surface elevation at the wave-maker is the key part of the present work. We apply a generation scheme based on the snake principle, where a phase lag exists for the elevation and velocity components between the neighbouring wave paddles. However, as the same in Chen (2017), a static domain boundary is applied, where only certain velocity is imposed to each virtual wave paddle. But the difference between the current scheme and the scheme used in Chen (2017) is that the velocity is not the oscillating velocity of the piston type wave-maker. Instead, the particle velocity of the generated plane waves from each wave paddle is used. Therefore, the evanescent modes are excluded. It is emphasized here that this approach is used to achieve a better agreement between the numerical and experimental results, as in the numerical model, the virtual wave paddles work in a similar way to the wave-maker in the physical experiments. The underlying requirements are that the same width at the wave-maker side and the same number of flaps as in the experiments should be used. However, if one does not aim to reproduce the experiments, but just to generate oblique waves, it is recommended to use the boundary conditions derived based on the surface elevation and velocity profiles from the oblique progressive waves.

\subsection{Surface elevation and particle velocity for the oblique focused wave groups}

This section derives the expressions of the surface elevation and velocity components for the oblique focused wave groups in the global coordinate system $\boldsymbol{x}=(x, y, z)$. Here we define the coordinate system $\boldsymbol{x}=(x, y, z)$ that $x$ direction is the longitudinal direction which is normal to the wave-maker side. $y$ direction is the transverse direction along the wave-maker side. $z$ direction is the vertical direction and gravity is acting along $-z$ direction. The origin of the coordinate system is located at the still free surface at the focal position. Meanwhile, a second coordinate system $\left(x^{\prime}, y^{\prime}, z^{\prime}\right)$ is also used in the derivation where $x^{\prime}$ direction is 


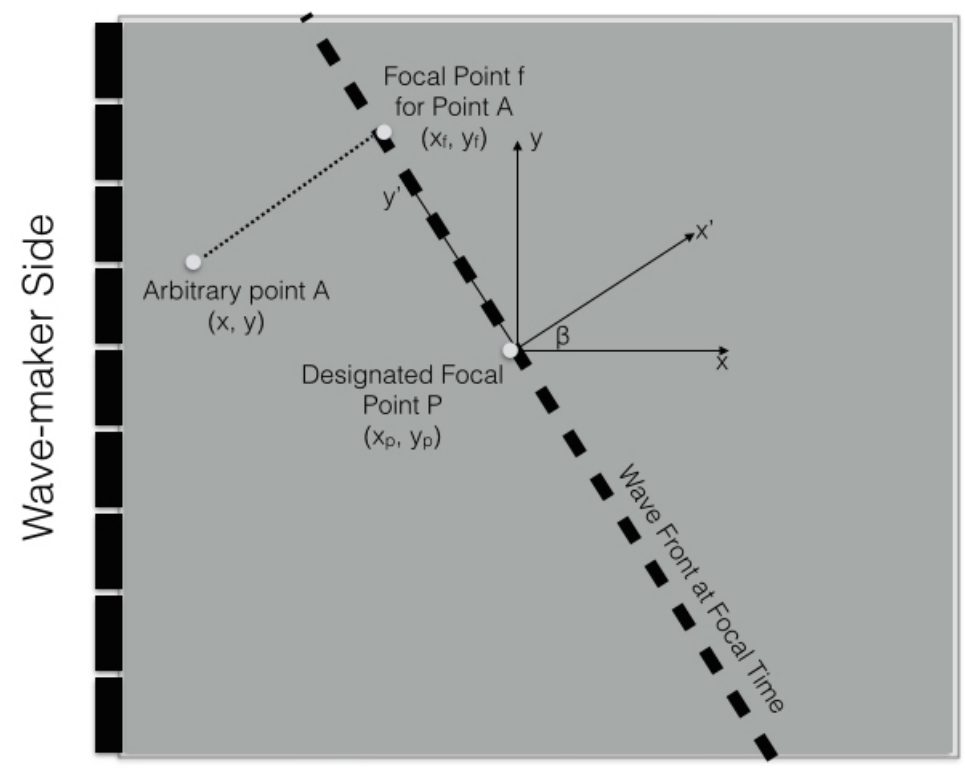

Figure 1: Illustration on the general configuration of focused wave propagation obliquely with an angle of $\beta$. The numerical model applies a Cartisan coordinate system of $(x, y, z)$, while $\left(x^{\prime}, y^{\prime}, z^{\prime}\right)$ is defined where $x^{\prime}$ axis is aligned with the direction of wave propagation. Point $\mathrm{P}\left(x_{p}, y_{p}\right)$ is the designated focal point. For an arbitrary point A with coordinate $(x, y)$, Point $\mathrm{f}$ is its corresponding focal point, whose coordinate is $\left(x_{f}, y_{f}\right)$.

aligned with the wave propagation direction. Therefore, the rotation angle between two coordinate systems is actually the wave propagation angle $\beta$.

Fig. 1 presents the general configuration of this problem with the defined two coordinate systems. Consider that a focused wave group is propagating obliquely with an angle of $\beta$ in the wave tank. Point $\mathrm{P}\left(x_{p}, y_{p}\right)$ is the desired focal point, where wave groups are supposed to be focused. This can be the place where the structure is located. Recall that for uni-directional focused wave groups, the wave front at focal time is actually a straight line, which is denoted as the dashed line passing Point $\mathrm{P}$ in Fig. 1. For an arbitrary point $\mathrm{A}$ with its coordinate $(x, y)$, its corresponding focal point is $\mathrm{f}$, whose coordinate is given as $\left(x_{f}, y_{f}\right)$.

As the starting point of the derivation, it is known that in $\left(x^{\prime}, y^{\prime}, z^{\prime}\right)$ coordinate system, the surface elevation $\eta^{\prime}$, for the uni-directional focused wave group for an arbitrary point $\left(x^{\prime}, y^{\prime}\right)$ at an arbitrary time $t$, is expressed as:

$$
\eta^{\prime}\left(x^{\prime}, t\right)=\sum_{i=1}^{N} a_{i} \cos \left[k_{i}\left(x^{\prime}-x_{f}^{\prime}\right)-\omega_{i}\left(t-t_{p}\right)+\epsilon_{i}\right]
$$

where $t_{p}$ is the focal time and $N$ is the number of wave components, $a$ is the wave amplitude, $\epsilon$ is the initial phase, $\omega$ is the angular frequency and $k$ is the wave number. Given that $\left(x^{\prime}, y^{\prime}\right)$ is obtained from $(x, y)$ by rotating $\beta$, the relation between two coordinate systems yields:

$$
x^{\prime}=x \cos \beta+y \sin \beta
$$

Substituting Eq. (2) into Eq. (1), the expression of the surface elevation in $(x, y, z)$ coordinate system reads:

$$
\eta(x, y, t)=\sum_{i=1}^{N} a_{i} \cos \left[k_{i} \cos \beta\left(x-x_{f}\right)+k_{i} \sin \beta\left(y-y_{f}\right)-\omega_{i}\left(t-t_{p}\right)+\epsilon_{i}\right]
$$

Eq. (3) has a similar form as the 3D wave focusing on a point. However, it should be mentioned that in Eq. (3), the position of the actual point $\left(x_{f}, y_{f}\right)$ is not fixed. For an arbitrary point $(x, y)$, its corresponding 
focal position is calculated as:

$$
\begin{aligned}
& x_{f}=\frac{1}{1+\tan ^{2} \beta}\left(x \tan ^{2} \beta-\left(y+y_{p}\right) \tan \beta+x_{p}\right) \\
& y_{f}=\tan \beta\left(x_{f}-x\right)+y
\end{aligned}
$$

The expressions for the velocity components can be derived in the same way, which are given below:

$$
\begin{aligned}
& u(x, y, z, t)=\sum_{i=1}^{N} a_{i} \omega_{i} \frac{\cosh \left(k_{i}(z+h)\right)}{\sinh \left(k_{i} h\right)} \cos \beta \cos \left[k_{i} \cos \beta\left(x-x_{f}\right)+k_{i} \sin \beta\left(y-y_{f}\right)-\omega_{i}\left(t-t_{p}\right)+\epsilon_{i}\right] \\
& v(x, y, z, t)=\sum_{i=1}^{N} a_{i} \omega_{i} \frac{\cosh \left(k_{i}(z+h)\right)}{\sinh \left(k_{i} h\right)} \sin \beta \cos \left[k_{i} \cos \beta\left(x-x_{f}\right)+k_{i} \sin \beta\left(y-y_{f}\right)-\omega_{i}\left(t-t_{p}\right)+\epsilon_{i}\right] \\
& w(x, y, z, t)=\sum_{i=1}^{N} a_{i} \omega_{i} \frac{\sinh \left(k_{i}(z+h)\right)}{\sinh \left(k_{i} h\right)} \sin \left[k_{i} \cos \beta\left(x-x_{f}\right)+k_{i} \sin \beta\left(y-y_{f}\right)-\omega_{i}\left(t-t_{p}\right)+\epsilon_{i}\right]
\end{aligned}
$$
below:

The amplitude of each component $a_{i}$ is calculated based on the target variance density spectrum as

$$
a_{i}=\frac{A}{m_{0}} \sum_{n=1}^{N} S_{n} \mathrm{~d} f
$$

which is a re-scale of the normalised auto-correlation function to the actual focal crest amplitude $A$. $S_{n}$ is the variance spectrum density and $m_{0}$ is the zero-th momentum of the spectrum. An empirical correction based on Li et al. (2018) is further employed to correct the amplitudes, in order to achieve better agreement with the experimental data. This correction scheme iteratively corrects the amplitude and phase of each component as below:

$$
\begin{gathered}
a_{i, \text { input }}^{k+1}=a_{i, \text { input }}^{k} \frac{a_{i, \text { target }}}{a_{i, \text { output }}^{k}} \\
\epsilon_{i, \text { input }}^{k+1}=\epsilon_{i, \text { input }}^{k}-\left(\epsilon_{i, \text { target }}-\epsilon_{i, \text { output }}^{k}\right)
\end{gathered}
$$

Here the $i$-th component of the amplitude at the $(k+1)$ th iteration $a_{i, \text { input }}^{k+1}$ is corrected by the ratio of the $i$-th component of the target amplitude $a_{i \text {,target }}$ and the $i$-th component of the output amplitude from the $k$-th iteration $a_{i, \text { output }}^{k}$. Meanwhile, the $i$-th component of the phase at the $(k+1)$ th iteration $\epsilon_{i, \text { input }}^{k+1}$ is corrected by the difference between the $i$-th target phase $\epsilon_{i, \text { target }}$ and the $i$-th component of the output phase from the $k$-th iteration $\epsilon_{i, \text { output }}^{k}$.

Fig. 2 presents an example of the surface elevation for an oblique phase-focused wave group using Eq. $(3)$ and Eq. (4). The wave group is generated based on JONSWAP spectrum (Hasselmann et al., 1973) with a focal crest of $22 \mathrm{~m}$. It is propagating obliquely with an angle of $25^{\circ}$ in a domain of ([-1000 $\mathrm{m} 1000$ $\mathrm{m}],[-1000 \mathrm{~m} 1000 \mathrm{~m}])$.

\subsection{Expressions at the wave-maker side for oblique focused wave groups}

The expressions at the wave-maker side for the oblique focused wave groups can be obtained by superposition of the expressions induced by its linear components. Appendix A presents a short overview of the derivation on the linear oblique progressive wave field in the basin with prescribed velocity on each wave paddle. The direction of the propagating waves is determined by the phase lag between the neighbouring paddles.

Given Eq. (3) as the target wave elevation, each component $\eta_{i}$ is given as:

$$
\eta_{i}=a_{i} \cos \left[k_{i} x \cos \beta+k_{i} y \sin \beta-\omega_{i} t+\epsilon_{i}^{\prime}\right]
$$

where $\epsilon_{i}^{\prime}=k_{i} x_{f} \cos \beta+k_{i} y_{f} \sin \beta-\omega t_{f}+\epsilon_{i}$. Therefore, such oblique wave component can be generated by a prescribed snake-type wave-maker motion. However, in the present work, instead of using the velocity of 

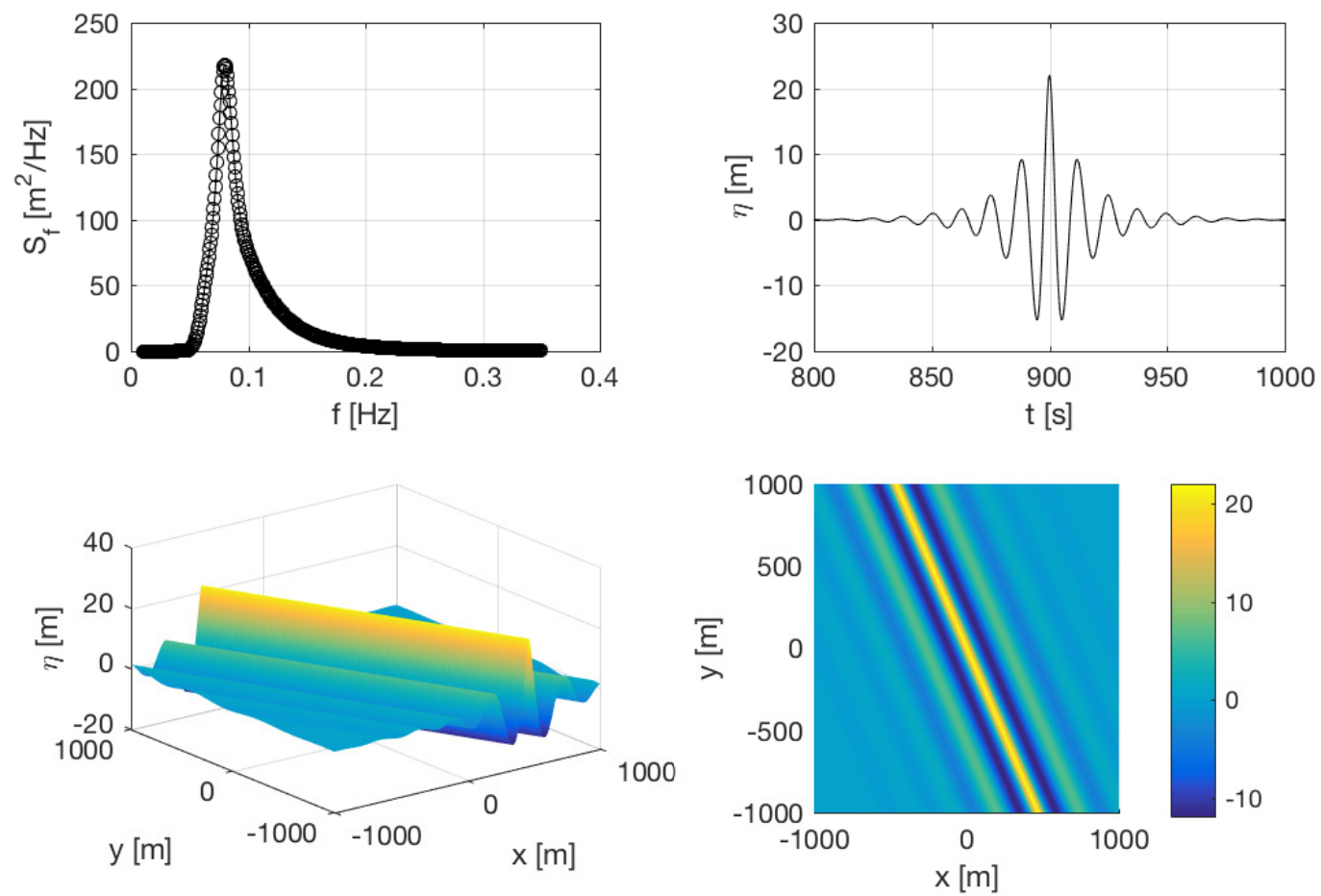

Figure 2: An example on the surface elevation of the oblique phase-focused wave group using Eq. 37 and Eq. (4). JONSWAP spectrum is applied with the peak period $T_{p}=12.5 \mathrm{~s}$, significant wave height $H_{s}=11.0 \mathrm{~m}$ and enhancement factor $\gamma=2.0$. The crest height at focal position $A=22 \mathrm{~m}$. The desired focal position is located $x_{p}=0, y_{p}=0$. The wave group is focused at $t=900 \mathrm{~s}$. The wave angle $\beta=25^{\circ}$ and water $\operatorname{depth} h=45 \mathrm{~m}$. Upper left: The applied JONSWAP spectrum. Upper right: The time series of surface elevation at focal point $x=0, y=0$. Lower Left: 3D bird view of surface elevation at focal time $t=900 \mathrm{~s}$. Lower right: top view of surface elevation at focal time $t=900 \mathrm{~s}$.

the wave-maker as the boundary condition, we applied the particle velocity of the plane progressive waves generated by the wave-maker. Therefore, given the coordinate of the wave paddle as $\left(x_{j}, y_{j}\right)$, the boundary conditions are written as:

$\eta_{j}(t)=\sum_{i=1}^{N} a_{i} \cos \beta \cos \left(k_{i} \cos \beta\left(x_{j}-x_{j, f}\right)+k_{i} \sin \beta\left(y_{j}-y_{j, f}\right)-\omega_{i}\left(t-t_{p}\right)+\epsilon_{i}\right) \quad j=1 \cdots M$

$u_{j}(z, t)=\sum_{i=1}^{N} a_{i} \omega_{i} \cos \beta \frac{\cosh \left(k_{i}(z+h)\right)}{\sinh \left(k_{i} h\right)} \cos \left(k_{i} \cos \beta\left(x_{j}-x_{j, f}\right)+k_{i} \sin \beta\left(y_{j}-y_{j, f}\right)-\omega_{i}\left(t-t_{p}\right)+\epsilon_{i}\right) \quad j=1 \cdots M$

$v_{j}(z, t)=0$

$w_{j}(z, t)=\sum_{i=1}^{N} a_{i} \omega_{i} \cos \beta \frac{\sinh \left(k_{i}(z+h)\right)}{\sinh \left(k_{i} h\right)} \sin \left(k_{i} \cos \beta\left(x_{j}-x_{j, f}\right)+k_{i} \sin \beta\left(y_{j}-y_{j, f}\right)-\omega_{i}\left(t-t_{p}\right)+\epsilon_{i}\right) \quad j=1 \cdots M$

It should be mentioned that there is an angle factor of $\cos \beta$ in Eq. 10 , which is due to the difference of the transfer function in the $2 \mathrm{D}$ and $3 \mathrm{D}$ cases. 


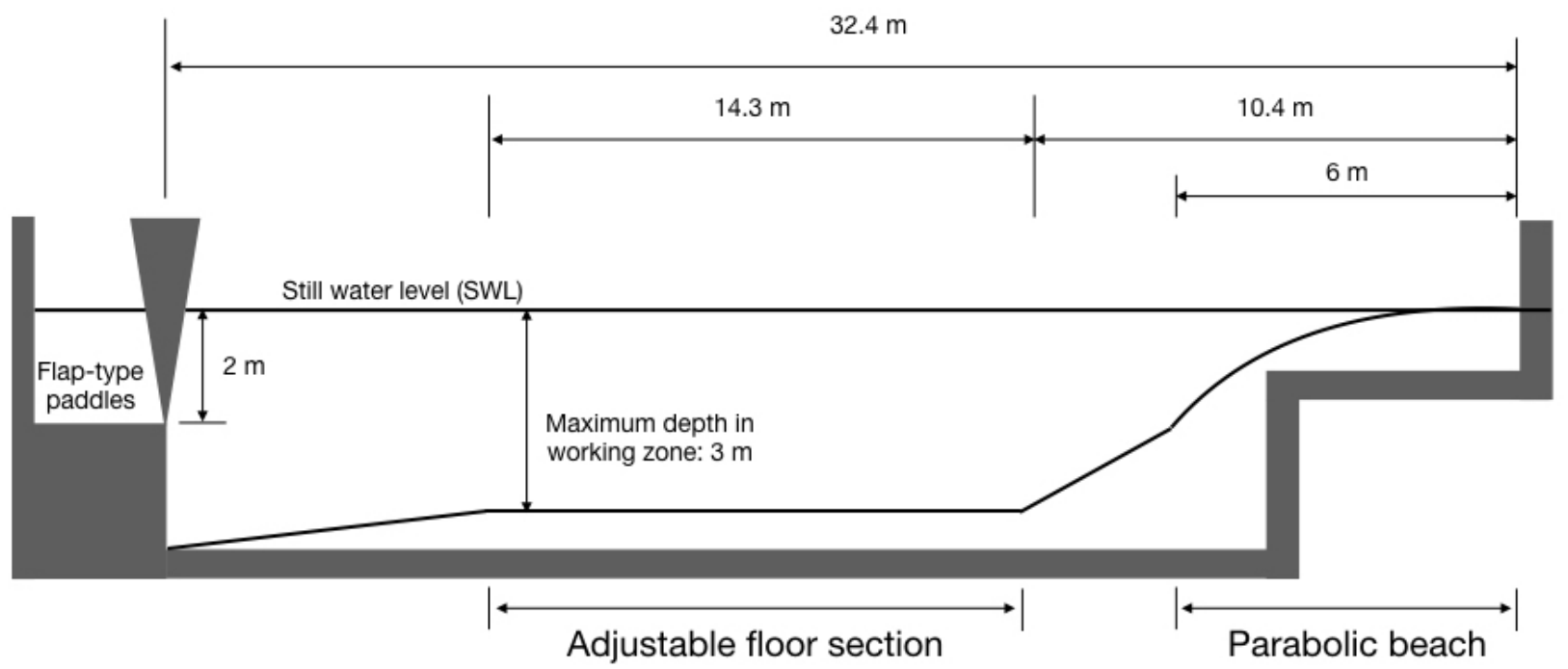

Figure 3: The dimensions and configuration of the Ocean Basin.

Table 1: The selected focused wave conditions for validation of the numerical model. $A$ is the focal crest amplitude, $T_{p}$ is the wave period for the wave component at peak frequency, $H_{s}$ is the significant wave height, $k_{p}$ is the wave number for the wave component at peak frequency and $\epsilon$ is the initial phase.

\begin{tabular}{cccccccc}
\hline Case & $A[\mathrm{~m}]$ & $T_{p}[\mathrm{~s}]$ & $H_{s}[\mathrm{~m}]$ & $h[\mathrm{~m}]$ & $k_{p} A[-]$ & $\epsilon[\mathrm{rad}]$ & $\beta\left[^{\circ}\right]$ \\
\hline 1 & 0.08930 & 1.456 & 0.103 & 2.93 & 0.13 & $\pi$ & 0 \\
\hline 2 & 0.08930 & 1.456 & 0.103 & 2.93 & 0.13 & $\pi$ & 10 \\
\hline 3 & 0.08930 & 1.456 & 0.103 & 2.93 & 0.13 & $\pi$ & 20 \\
\hline
\end{tabular}

\section{Results and discussions}

\subsection{Experiments}

A set of dedicated experiments were performed at the Ocean Basin, COAST Laboratory in Plymouth University. The data is used to be compared with our numerical results. The dimension of the wave tank is $35 \mathrm{~m}$ long and $15.5 \mathrm{~m}$ wide. At the wave-maker side, there are 24 flap-type feedback controlled wave paddles with a hinge depth of $2 \mathrm{~m}$. The water depth at the wave-maker is $4 \mathrm{~m}$ and there is a linear slope connecting to the bottom at the working area. The depth at the working area is adjustable with a maximum of $3 \mathrm{~m}$ and it was set to $2.93 \mathrm{~m}$ in present experiments. The dimensions and configuration of the basin is presented in Fig. 3. The 24 flap type paddles can produce regular waves with a maximum height of $0.9 \mathrm{~m} \mathrm{at} 0.4 \mathrm{~Hz}$ and wave height above $0.2 \mathrm{~m}$ in a range of $0.166 \mathrm{~Hz}-1 \mathrm{~Hz}$. Oblique waves can be generated up to $40^{\circ}$ from the normal direction.

The selected test conditions are given in Table 1, which have the same steepness but with different propagation angles. Essentially, these waves were generated by using the linear superposition of 244 wave fronts with frequencies evenly spaced between $0.1 \mathrm{~Hz}$ and $2 \mathrm{~Hz}$ and a theoretical focus location of $13.886 \mathrm{~m}$ from the wave paddles. The amplitudes of the frequency components were derived by applying the NewWave theory to a JONSWAP spectrum with the parameters in Table 1

Totally two sets of experiments were carried out, one set for focused wave groups propagating in the empty tank, and the other with an FPSO-shaped body in place. The FPSO model was made of aluminium and fabricated at 1:100 scale comprising of a rectangular box and a half circular cylinder at the bow and the stern. The height and width of the model were $0.3 \mathrm{~m}$ and length of the model was $1.2 \mathrm{~m}$. It was initially half immersed in the still water and rigidly fixed to the gantry, which spanned the width of the Ocean Basin. In both sets of experiments, the surface elevation were measured by the resistance type wave gauges with a sampling frequency of $128 \mathrm{~Hz}$. The positions of the wave gauges are given in Fig. 4 and Fig. 5, for each set 


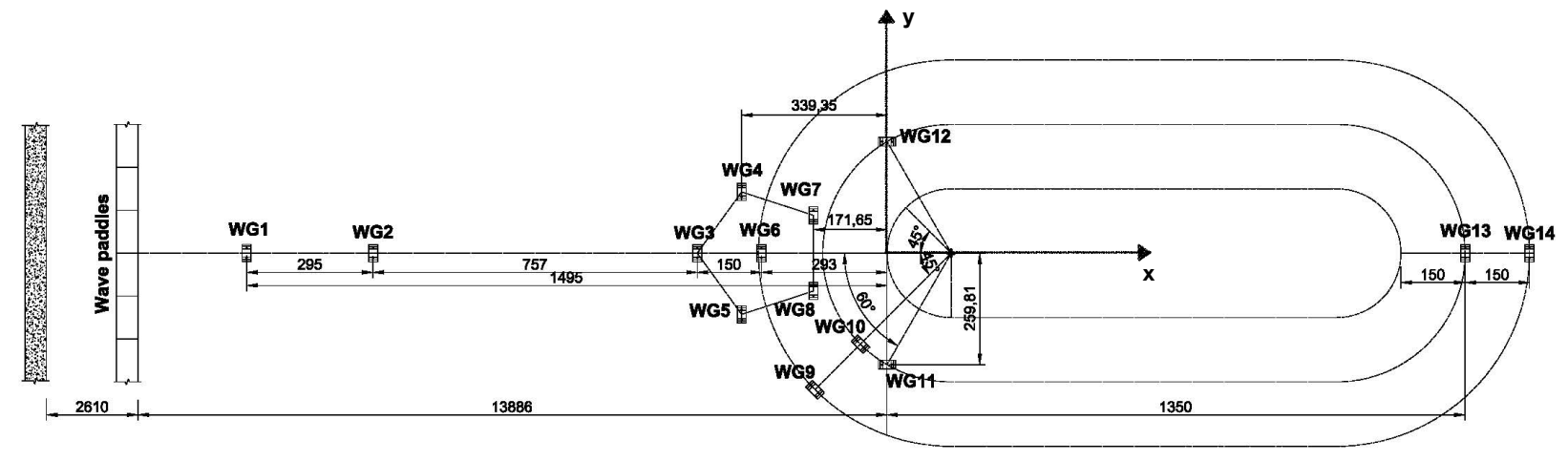

Figure 4: Top view (x-y plane) of the positions of the wave gauges for the cases with oblique phase-focused wave groups propagating in the empty wave tank (unit: $\mathrm{mm}$ ).

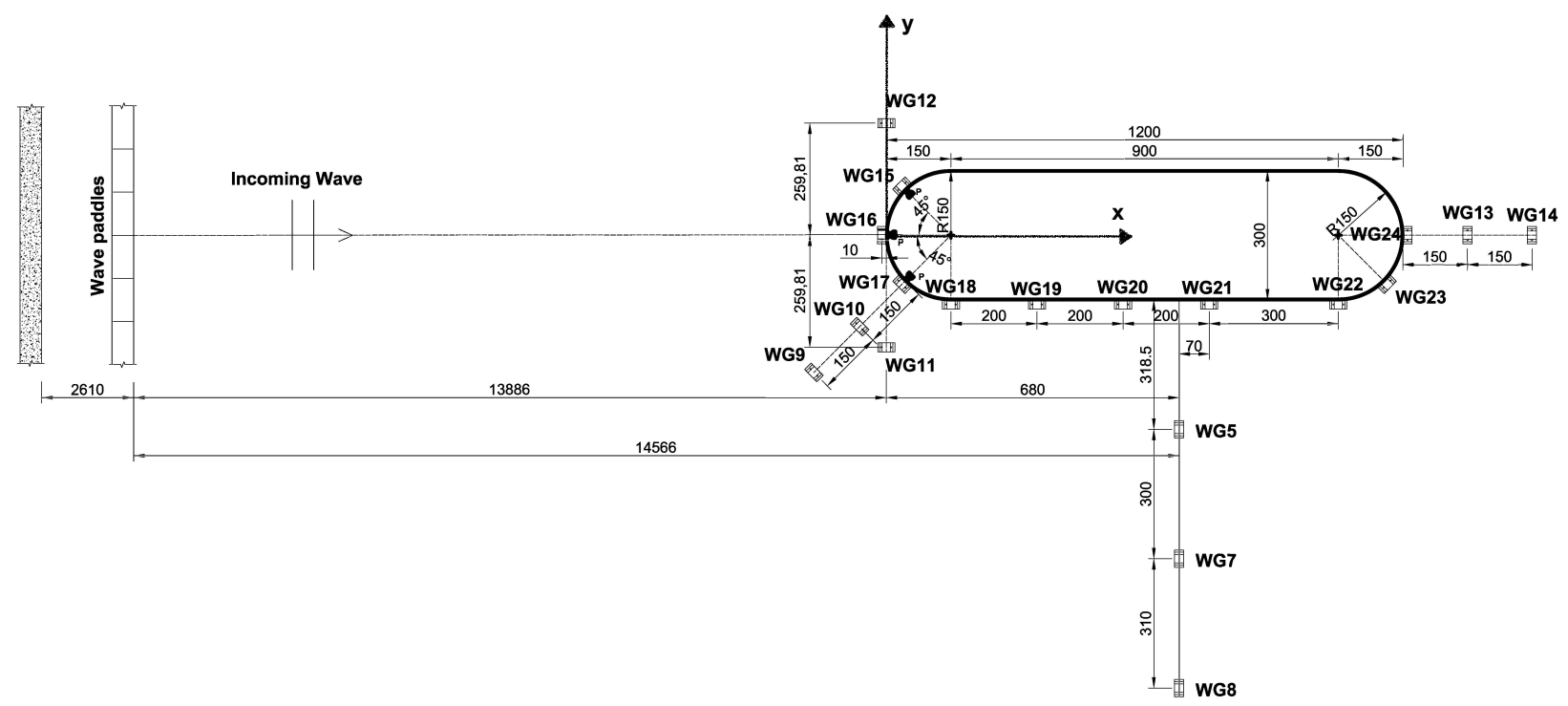

Figure 5: Same as in Fig. 4 but for the cases with FPSO in place.

of experiments, respectively. Meanwhile, for the second set of experiments, the local pressure on the FPSO was also measured at nine positions on the surface of the bow of the FPSO, as shown in Fig. 66. Readers are referred to Mai et al. (2016) for further details on the setup of the experiments.

\subsection{Numerical model}

\subsubsection{Governing equations}

The present numerical model solves the incompressible Navier-Stokes equations for a two-phase flow of water and air with incorporation of a volume of fluid (VOF) scheme to capture the free surface (Hirt and Nichols, 1981). The governing equations include conservation equations for the mass and momentum plus a transport equation for the volume fraction of water in the Eulerian frame of reference as shown below:

$$
\begin{gathered}
\frac{\partial u_{i}}{\partial x_{i}}=0 \\
\frac{\partial \rho u_{i}}{\partial t}+\frac{\partial \rho u_{j} u_{i}}{\partial x_{j}}-\frac{\partial}{\partial x_{j}} \mu\left(\frac{\partial u_{i}}{\partial x_{j}}+\frac{\partial u_{j}}{\partial x_{i}}\right)=-\frac{\partial p^{*}}{\partial x_{i}}-g_{j} x_{j} \frac{\partial \rho}{\partial x_{i}}
\end{gathered}
$$




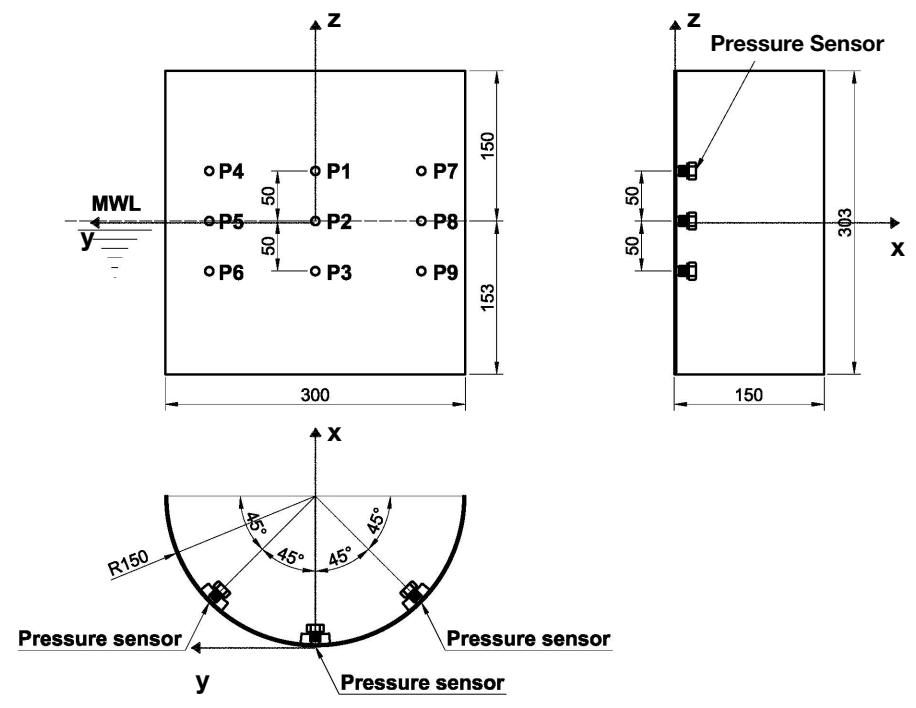

Figure 6: The layout of the pressure sensor on the surface of the bow of the FPSO.

$$
\frac{\partial \alpha}{\partial t}+\frac{\partial}{\partial x_{i}}\left(u_{i} \alpha\right)+\frac{\partial}{\partial x_{i}}\left(u_{i}^{r} \alpha(1-\alpha)\right)=0
$$

where $x_{i}=(x, y, z)$ is the Cartesian coordinate system, which is the same as used in Section $2, u_{i}=(u, v, w)$ is the velocity component, $g_{i}=(0,0,-9.81)$ is the gravity component, $\rho$ is the density, $\mu$ is the viscosity and $p^{*}=p-\rho g_{i} x_{i}$ is the dynamic pressure, as the coordinate system is defined that $z=0$ corresponds to the still free surface. Introducing $p^{*}$ can ease the definition of the boundary condition, while the total pressure $p$ is reconstructed at each time step when $p^{*}$ is solved. $\alpha$ is the water volume fraction, where $\alpha=1$ gives a cell filled by water and $\alpha=0$ represents a cell with air. The interface lies between $0<\alpha<1$. $u_{i}^{r}$ here is referred as the compressive velocity component (Berberović et al., 2009), which aids in retaining a sharp interface. The term $\alpha(1-\alpha)$ vanishes everywhere except at the interface.

\subsubsection{Discretization schemes and algorithms}

This set of equations are discretized and solved within the framework of the open source CFD-toolbox OpenFOAM ${ }^{\circledR}$. Finite volume method is employed to solve them with a collocated variable arrangement. The time derivative terms are discretised using a first-order implicit Euler scheme, while the convection terms are discretised using TVD (Total Variation Diminishing) schemes, i.e. a blend of central difference and upwind schemes, depending on the ratio of the successive gradients. They are globally second order, although locally can be reduced to the first order. The Laplacian operator is discretised using the linear differencing scheme, which is further corrected to account for the mesh non-orthogonality. The applied schemes are presented in Table 2 in details, for the convenience of the readers to reproduce the simulations. The inner-coupling of pressure and velocity is resolved by using PISO (Pressure Implicit with Splitting of Operators) algorithm (Issa, 1986), where Rhie-Chow interpolation is implicitly implemented in the present solver to avoid the pressure-velocity decoupling due to the segregated solution of momentum and mass-conservation equation when using a collocated discretisation (Rhie and Chow, 1982). 
Table 2: The employed discretisation scheme for each term in Eq. 11, Eq. 12, and Eq. 13.

\begin{tabular}{ccc}
\hline Term & $\begin{array}{c}\text { Applied schemes } \\
\text { in OpenFOAM }\end{array}$ & Brief explanation \\
\hline$\frac{\partial}{\partial t}$ & - & First order explicit Euler scheme \\
$\left.\frac{\partial x_{i}}{\partial t} u_{i} \alpha\right)$ & MUSCL & Monotonic Upwind Scheme for Conservation Laws \\
$\frac{\partial}{\partial x_{i}}\left(u_{i}^{r} \alpha(1-\alpha)\right)$ & interfaceCompression & $\begin{array}{c}\text { A special designed TVD scheme } \\
\text { for interface compression term }\end{array}$ \\
$\frac{\partial \rho u_{i}}{\partial t}$ & Euler & First order implicit Euler scheme \\
$\frac{\partial \rho u_{j} u_{i}}{\partial x_{j}}$ & limitedLinearV 1 & $\begin{array}{l}\text { Linear scheme that limits towards upwind } \\
\text { in regions of rapid changing gradient. }\end{array}$ \\
$\frac{\partial}{\partial x_{j}} \mu\left(\frac{\partial u_{i}}{\partial x_{j}}+\frac{\partial u_{j}}{\partial x_{i}}\right)$ & linear corrected & $\begin{array}{l}\mu \text { an from centre differencing scheme, } \\
\text { with explicit non-orthogonal corrections for } \nabla \boldsymbol{u}\end{array}$ \\
\hline
\end{tabular}

\subsubsection{Model setup}

This section introduces the configuration of the numerical model. Prior to set up the final numerical model, a series of numerical experiments are firstly conducted to examine the sensitivity of the numerical solution on the domain width, paddle number at the wave-maker side, the computational mesh and the time step, which are shown in Appendix B. The brief conclusions are that (1) The width of the paddle is recommended to be smaller than $0.33 L$, where $L$ is the characteristic wave length. (2) The length of the wave-maker side should be larger than 6.58L. (3) Regarding the mesh resolutions, four different mesh resolutions are tested as shown in Table 3 for focused wave group propagating in the empty wave tank normal to the wave-maker. Please refer to Table 3 for the detailed parameters related to the different mesh resolutions. We are not able to achieve a really converged numerical solution even if the total mesh cells reach 18.26 million. The difference of the surface elevation at the focal time between the finest two resolutions is around 7\%. (4) Four different Courant number limits are also tested, and the numerical results are unlikely to be affected by the time step as long as the Courant number limit is below 0.35 . Readers are referred Table 4 for detailed parameters related to the time step convergence test.

In the present work, in order to reproduce the experiments, we use exactly the same tank width and depth as in the experiments. But the length of the tank is shortened to $10 \mathrm{~m}$ long, to reduce the computational cost. Meanwhile, the paddle number is also the same, namely 24 virtual paddles are applied. Such a configuration also satisfy the requirements shown above. The maximum Courant number that is allowed in the simulation is 0.25 , which can produce a numerical solution that is independent of time step. Regarding the mesh, in order to achieve a computationally affordable model, we choose to use Grid 3, as shown in Table 3, for oblique wave propagating in the empty wave tank. Such a grid resolution makes a reasonable compromise between the accuracy of the numerical solution and the computational cost. For the case with FPSO in place, this is also used as the background mesh. Three levels of refinement is further performed close to the FPSO in $x$ and $y$ directions. This ensures a nearly unit aspect ratio for the mesh cells in the vicinity of the FPSO, which is optimal for the application of the utility snappyHexMesh, where the background mesh eventually conforms to the surface by iteratively refining the initial background mesh and morphing the resulting split-hex mesh to the surfaces. Furthermore, significant free surface deformation may arise near the FPSO, which is better resolved by the refined mesh. An example of the snapshot of the computational mesh near the FPSO is given in Fig. 7.

The waves are generated based on the same target spectrum, and the surface elevation and velocity profiles are superposed by 244 components in the same frequency interval. Essentially they should focus at $t_{f}=8 \mathrm{~s}$ based on the linear dispersion relation. The amplitudes and initial phase of these components are further corrected based on the correction scheme introduced in Eq. (7) - 8). Regarding the boundary conditions used for the numerical wave tank, the inlet condition has been presented in Section 2 Active absorption boundary presented in Higuera et al. (2013) is used as the outlet boundary condition, which can enhance the stability of the system by decreasing the energy and correcting the increasing water level 


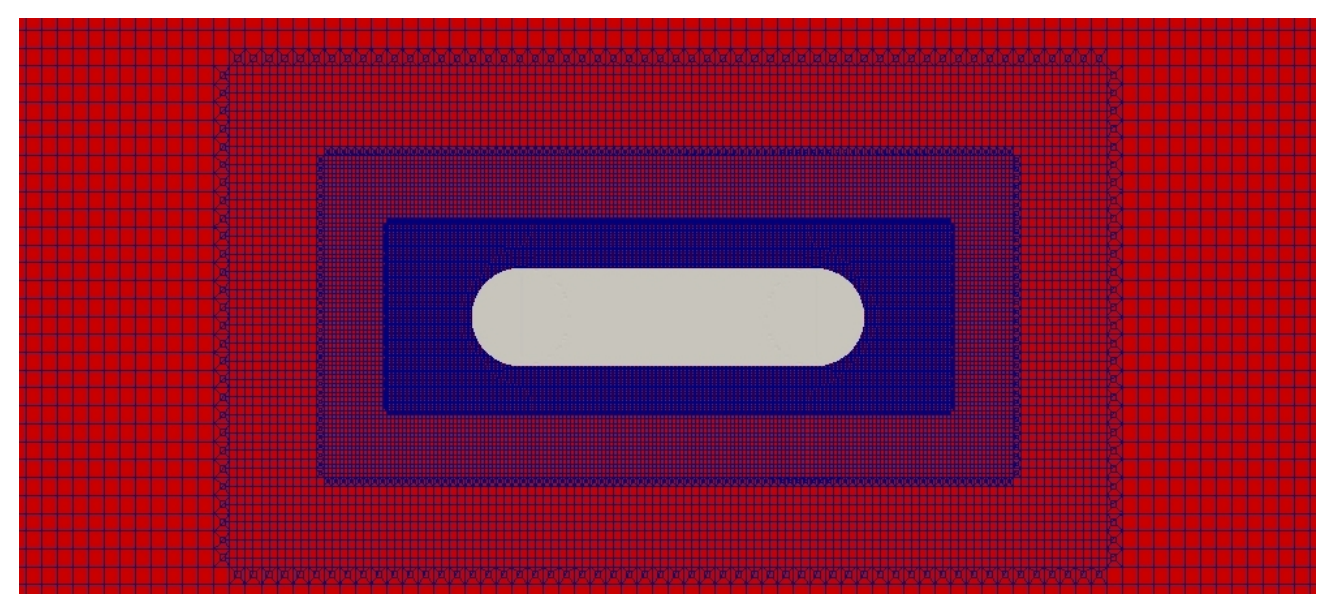

Figure 7: The computational mesh near the FPSO cylinder.

in long simulations. Meanwhile, it does not increase the computational cost. Hereby it is preferred in the present work. Atmospheric condition is used on the top of the wave tank, where the velocity is set to the so-called pressurelnletOutletVelocity, a native boundary condition supplied with OpenFOAM, which applies zero gradient on all components of the velocity except where there is inflow, in which case a fixed-value condition is applied. The pressure boundary condition is set to totalPressure. This means when there is reversed inflow, the pressure needs to be reduced by subtracting the dynamic pressure $1 / 2 \rho|u|^{2}$. We also test the difference of the solutions when using reflective and absorptive wall as the side wall condition, when is shown in Appendix B. Essentially it is found that the difference is visually negligible. Therefore, we just choose absorptive side wall, i.e. the same boundary condition as used in the outlet.

\subsection{Modelling of obliquely propagating focused wave groups}

\subsubsection{Surface elevation}

The surface elevation contour at the theoretical focal time $t_{f}$ is given in Fig. 8. When $\beta=0^{\circ}$, the incident wave group is normal to the wave-maker, and the flux injected into the computational domain are identical for all the wave paddles. Therefore, the side wall and limited tank width do not affect the wave propagation, and the generated waves cover all the domain. However, when $\beta$ is increasing, the side wall and limited tank width start to affect the generated waves. In the lower part, a shadow area due to the limited tank width appears where the wave amplitudes are significantly reduced. The area of this shadow area is dependent on the propagation angle $\beta$. Similar phenomenon have also been observed in O'Dea and Newman (2007), based on the linear potential solver WAMIT. In the upper part, we still notice the form of standing waves due to reflection, as the absorbing boundary did not absorb all the wave energy that is perpendicular to the side wall perfectly, especially for such case where the ambient angle of the propagating wave is rather large for the side wall. However, in the central part far away from the side wall, a uniform wave front is formed, which is the effective area for the model test. It is of difficulty to give an exact solution on the range of the effective working area. However, it can be roughly estimated as shown in Fig. 8 for the area between the black lines, which is determined by the propagation angle. One can see that with the increasing propagation angle, the area is rapidly reduced.

We present comparison of the surface elevation in Fig. 9 at a number of wave gauges ranging from the field near the wave-maker to the outlet area for the cases of three directional waves propagating in the empty wave tank. Meanwhile, a zoom-in of the comparison at Gauge 11 (the theoretical focal position for the normal wave group) is given in Fig. 10. For $\beta=0^{\circ}$, the average discrepancy between the numerical solution and the experimental data is $7 \%$, taken the experimental data as the reference. The discrepancy should be mainly due to the reason that the numerical model does not consider the shoaling process, as occurred in the physical wave tank due to the elevation of the bottom (see Fig. 3). The wave-maker 

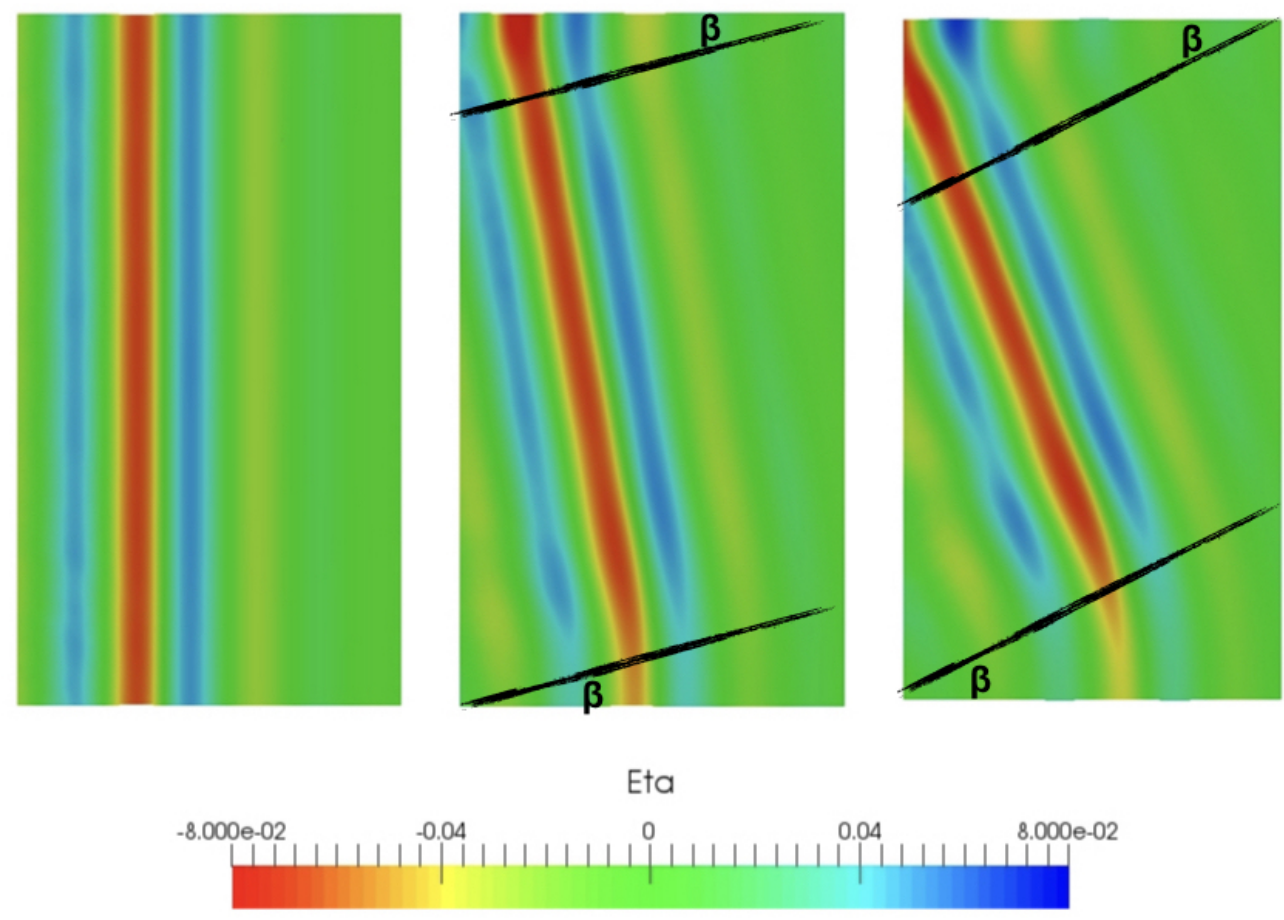

Figure 8: The surface elevation contour at the theoretical focal time for the three cases $\beta=0^{\circ}$ (left), $\beta=10^{\circ}$ (middle) and $\beta=20^{\circ}$ (right). The black lines in the middle and right figure are the boarder of the estimated area that is not affected by the side wall effect. The unit shown in the figure legend for surface elevation Eta is meter.

signal was corrected to minimize the shoaling effect, but it was unknown to the authors, as the signal of the displacement was not released to the authors. The correction scheme in the numerical wave-making, as shown in Eq. (7)- 8), reduces the difference to some extent, but is not able to completely remove the discrepancy. Furthermore, the simulated JONSWAP spectrum has a relatively long tail with low and high frequency components. As the domain length in the numerical model is shorter than in the experiments, these components are prohibitively difficult to capture by the model. However, we should also emphasize that the experiments can neither capture all the components, considering the very low cut-off frequency.

As the incident angle $\beta$ increases, the effect of finite width of wave-maker is also introduced in both the numerical model and the experiments. This generally violates the assumption of the snake theory, where the wave-maker side is infinitely long and radiation boundary condition is typically assigned for the side walls. Though the configuration of the numerical model is very much similar to the experiments (same wave paddle number, wave-maker width, cut-off frequency and spectrum component), certain differences of the surface elevation are still observed, especially for the case with $\beta=20^{\circ}$, which reaches $15 \%$. The reasons are credited to the difference on the wave-making. We typically assign the surface elevation and the velocity based on the progressive plane wave generated by each paddle. However, in physical experiments, segmented wave paddles are moving based on the prescribed displacement. This is accompany with the local diffraction effect and water leakage between paddles. These effects are likely to influence the wave-making and result in the discrepancies.

\subsubsection{Free surface evolution}

The evolution of the free surface along its propagation direction is given in Fig. 11, which shows the dispersive focusing and defocusing process. The evolution process including both focusing and de-focusing is quite similar between the three cases, indicating that the angle has minor effect on the evolution. Due to the high order wave-wave interactions (predominantly by third order interaction effect (Ning et al. 2009$)$ ), the 

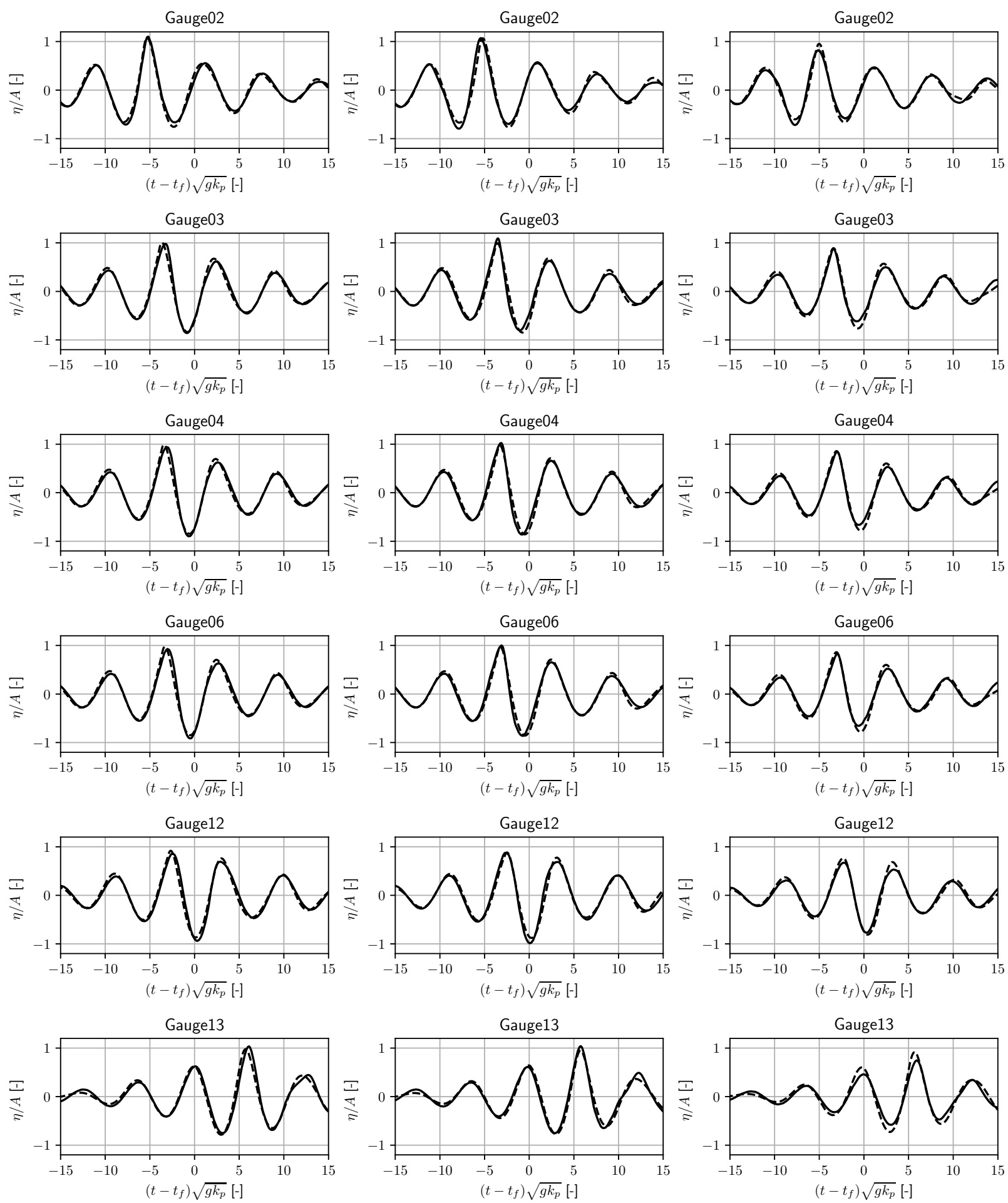

Figure 9: Comparison of the surface elevation at a variety of gauges between the numerical and experimental results, which are for the case of oblique focused wave groups propagating obliquely in an empty wave tank. The propagating angle $\beta=0^{\circ}$ (left), $\beta=10^{\circ}$ (middle), $\beta=20^{\circ}$ (right). Solid line: experimental results. Dashed line: numerical results from OpenFOAM. 

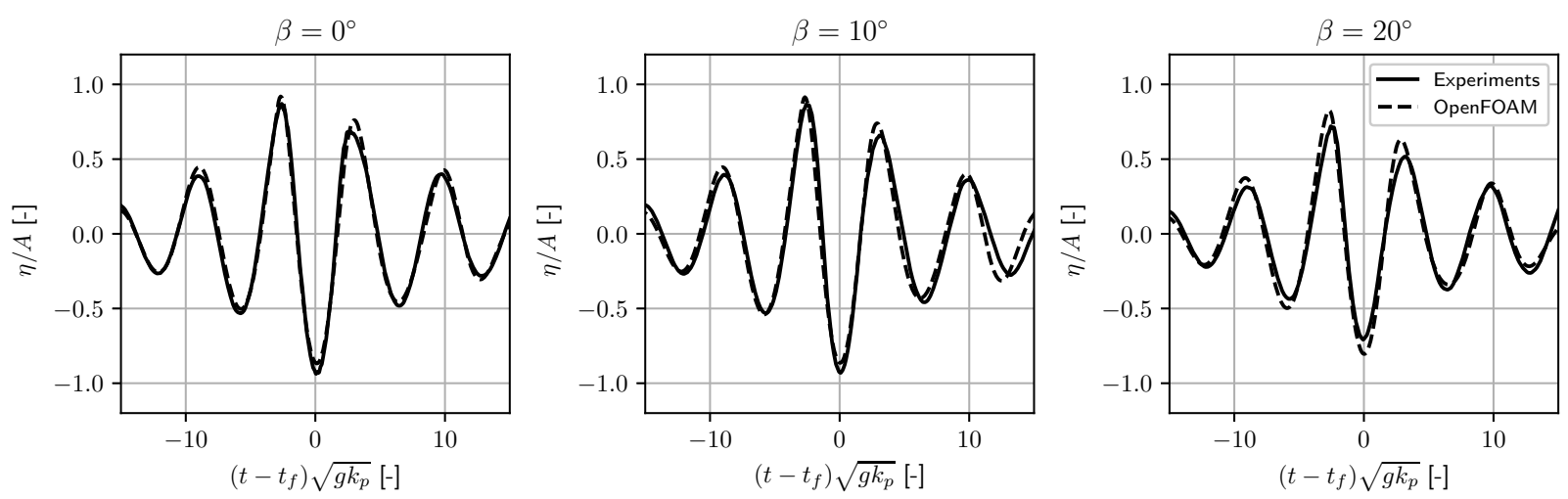

Figure 10: Detailed comparison of the surface elevation at Gauge 11 for the cases of oblique focused wave groups propagating in an empty wave tank under different angles. Dashed line: numerical results from OpenFOAM. Solid line: experimental results.

actual focal time and position are downshifted. However, the downshift time and distance are approximately identical for all the three cases, irrespective of the wave angle. This further indicates that the harmonic structures of the wave groups are kept almost the same, which motivates the harmonic analysis below in Section 3.3 .3 ,

\subsubsection{Harmonic analysis}

In order to further analyse the effect of wave angle on the harmonic structure of the generated waves, we separate the higher order harmonics using the phase-inversion method presented in Fitzgerald et al. (2014). The idea is that for focused wave groups, the crests of all the linear components coincide at the focal event. Hereby significant nonlinearity is only confined to the neighbourhood of the focal location around the focal time, where the envelope of the elevation can be regarded as a slow varying function of time $A(t)$ relative to the peak frequency component $\omega_{p}$. Therefore, the elevation is expanded in a way similar to regular waves:

$$
\eta(t)=B_{11} A(t) \cos \varphi_{p}+A^{2}(t)\left(B_{20}+B_{22} \cos \left(2 \varphi_{p}\right)\right)+A^{3}(t)\left(B_{31} \cos \varphi_{p}+B_{33} \cos \left(3 \varphi_{p}\right)\right)+\mathcal{O}(4)
$$

where $\varphi_{p}=\omega_{p} t+\epsilon . B_{i j}$ is the coefficients for the super-harmonics and sub-harmonics.

Given the harmonic structure of the surface elevation in Eq. 144, the first three harmonics can be separated via the linear combination as below:

$$
\begin{aligned}
& \frac{1}{4}\left(\eta_{0}-\mathrm{H}\left(\eta_{90}\right)-\eta_{180}+\mathrm{H}\left(\eta_{270}\right)\right)=B_{11} A(t) \cos \varphi_{p}+B_{31} A^{3}(t) \cos \varphi_{p}+\mathcal{O}\left(A^{5}(t)\right) \\
& \frac{1}{4}\left(\eta_{0}-\eta_{90}+\eta_{180}-\eta_{270}\right)=B_{22} A^{2}(t) \cos \left(2 \varphi_{p}\right)+B_{42} A^{4}(t) \cos \left(2 \varphi_{p}\right)+\mathcal{O}\left(A^{6}(t)\right) \\
& \frac{1}{4}\left(\eta_{0}+\mathrm{H}\left(\eta_{90}\right)-\eta_{180}-\mathrm{H}\left(\eta_{270}\right)\right)=B_{33} A^{3}(t) \cos \left(3 \varphi_{p}\right)+\mathcal{O}\left(A^{5}(t)\right)
\end{aligned}
$$

where $\mathrm{H}$ is the operator of Hilbert transformation for a given signal.

Fig. 12 reveals the harmonic structure of the focused wave groups under different propagation angles. In this case, the linear component is dominant over the higher order harmonics, which is around $88 \%$ of the amplitude crest $A$. We observe an excellent match of the first order harmonic for all the three cases. We notice a deviation of the second order harmonic at trough at $t^{\prime}=-0.18$, where $t^{\prime}=\left(t-t_{f}\right) \sqrt{g k_{p}}$ is the non-dimensional time. However, the peak amplitude at $t^{\prime}=1.16$ remains a good agreement with deviation of less than $1 \% A$. The same phenomenon is observed in the third order harmonic, where significant deviations are presented at $t^{\prime}=-0.58$. The deviations on the peak of the third order harmonic at $t^{\prime}=1.16$ is $0.2 \% A$, given that the magnitude of the third order harmonic itself is very small, i.e. less than $2 \% \mathrm{~A}$. 

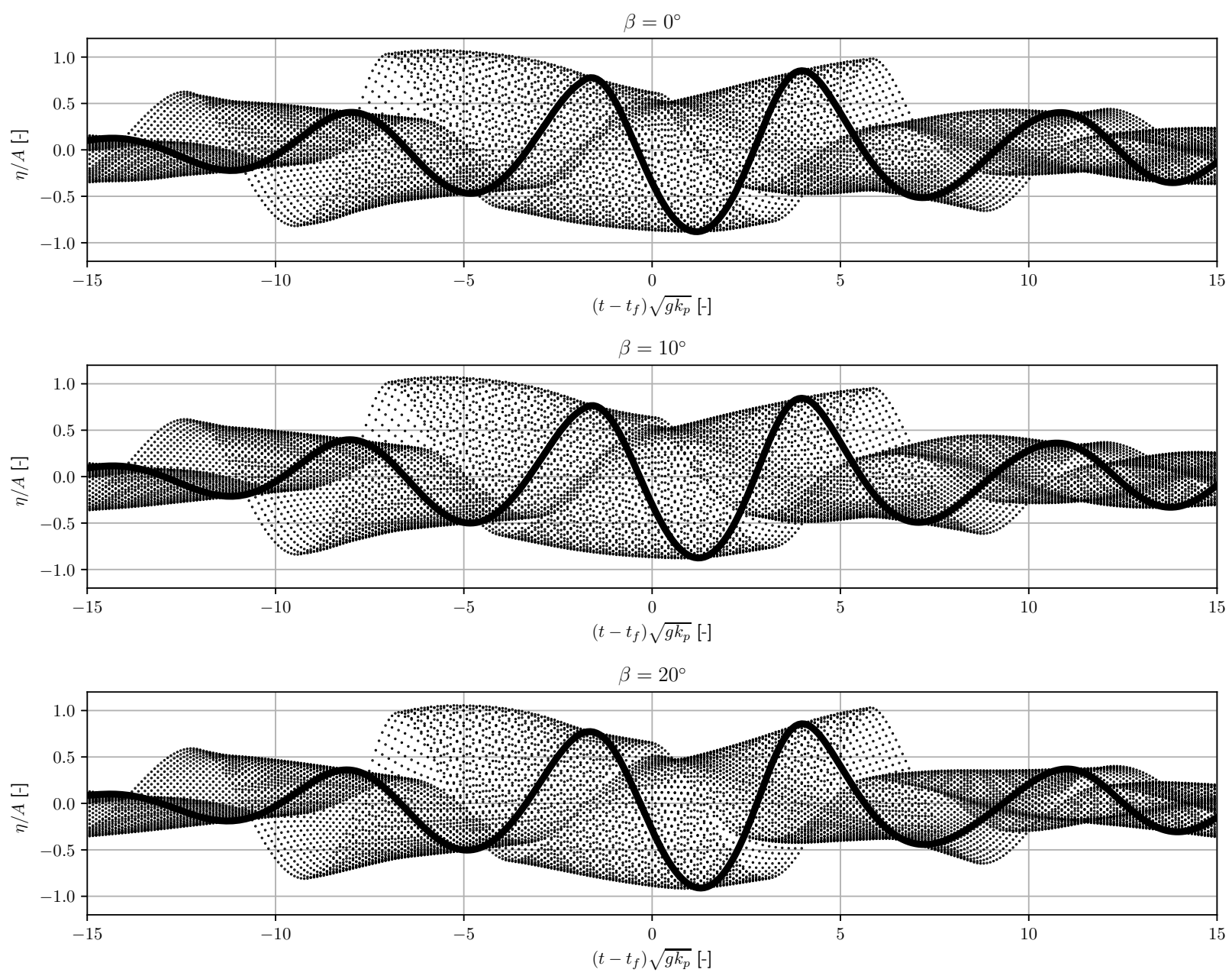

Figure 11: Free surface elevations at a series of gauges along the wave propagation direction passing the focal point. The wave gauges are distributed evenly with a distance of $0.1 \mathrm{~m}$ from the starting point of $2 \mathrm{~m}$ in front of the theoretical focal position to the end point of $1.4 \mathrm{~m}$ behind the theoretical focal position. The bold solid lines indicate the elevation where the wave group is focused.

\subsection{Modelling of oblique wave interaction with a fixed FPSO-shaped body}

\subsubsection{Surface elevation}

The comparison of the surface elevation at the selected wave gauges is presented in Fig. 14. We notice that the discrepancy between the numerical and the experimental results is likely to increase, comparing to the case with empty wave tank, especially at the gauges at the FPSO bow where the deviation can be up to $16 \%$ for $\beta=0^{\circ}$. Moreover, due to the side wall effect, the discrepancy is likely to grow with the increase of the propagation angle, where the numerical model tends to overestimate the surface elevation consistently.

We emphasize that the flow at the bow can be violent, as the trough is focused at that area. A snapshot of the flow near the FPSO is presented in Fig. 13. As can be seen, the incident wave group interacts with the FPSO and partially reflected. The reflected waves further interact with the incident wave group and a small concentric wave front is formed in front of the bow. This is the Type- 1 diffracted wave as defined in Swan and Sheikh (2015). However, at Gauge 24 which is located at the stern of the FPSO, the agreement is much better.

Furthermore, the discrepancy can be due to the reason that the wave gauge may not be exactly in the same place as in the experiments. The gauge was installed almost at the surface of the FPSO in the 

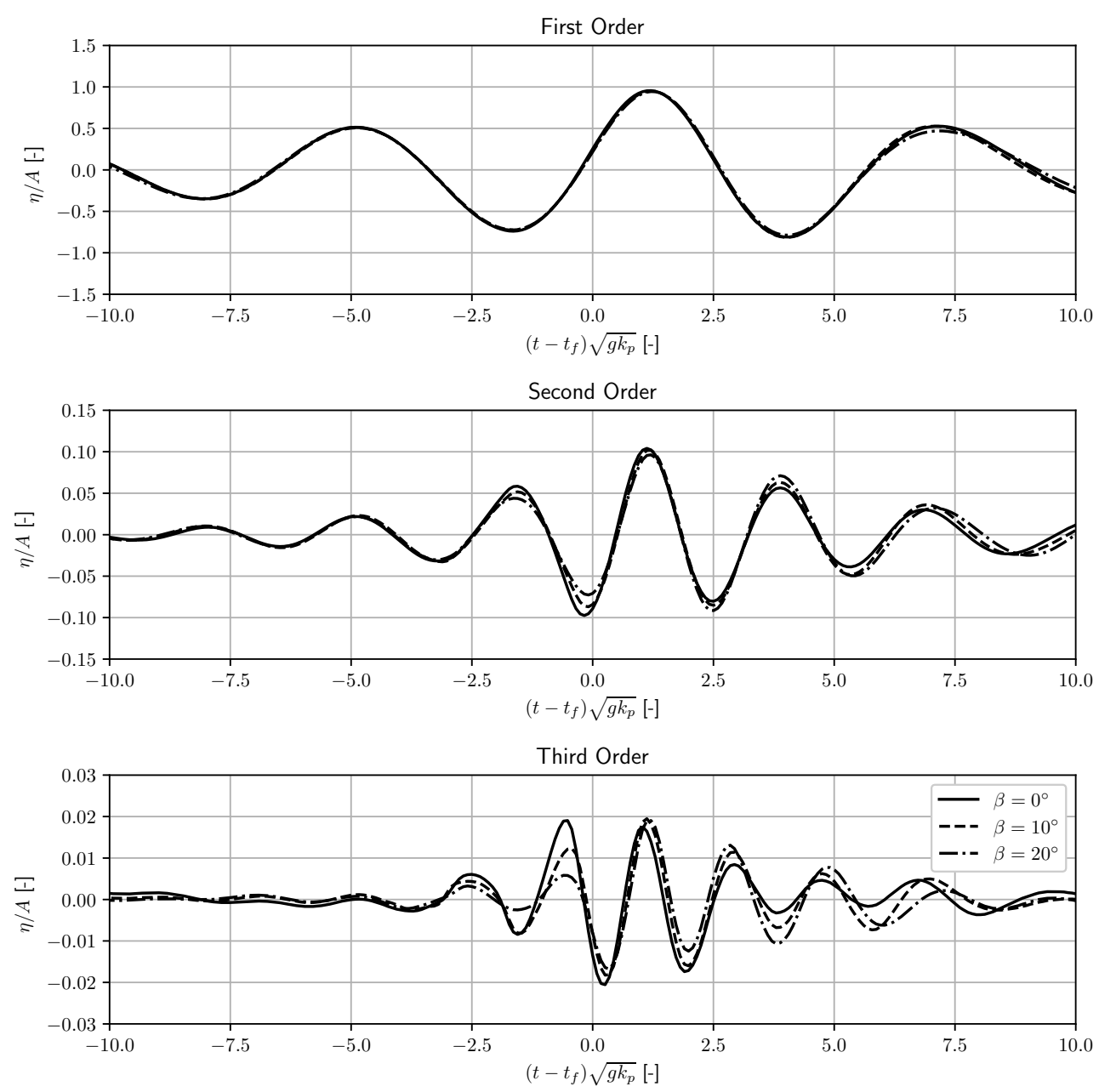

Figure 12: The extracted first, second and third order harmonics based on the phase-inversion method for the cases with different propagation angles.

laboratory test. However, in the numerical model, the elevation is not extracted from the boundary patch of the FPSO, which is not straightforward to extract and do the post-processing. We take the elevation from the numerical gauges at a few millimeters away from the surface. This can potentually introduce certain differences, especially where violent flow appears and substantial changes can occur within small areas.

\subsubsection{Local pressure}

The comparison of the local pressure is given in Fig. 15. The main discrepancy occurs at Sensor 02, which are installed at the still free surface. Therefore, the pressure is largely induced by the hydrodynamic effect. The overprediction of the second peak at $\left(t-t_{f}\right) \sqrt{g k_{p}} \sim 2.5$ is also consistent with the overprediction of the free surface elevation at Gauge 16. This indicates that the angle effect is under estimated by the numerical model. On the other hand, as Sensor 03 and 06 are initially submerged below the free surface, the hydrostatic pressure also contributes to the total pressure. As hydrostatic pressure is should be captured more accurately by the numerical model, the discrepancy is relatively small.

\subsubsection{Integrated wave forces on the FPSO}

The integrated forces on the FPSO is shown in Fig. 16. As the forces were not measured in the experiments, only numerical results are given here. From Fig. 16, the effect of incident angle is quite clearly 


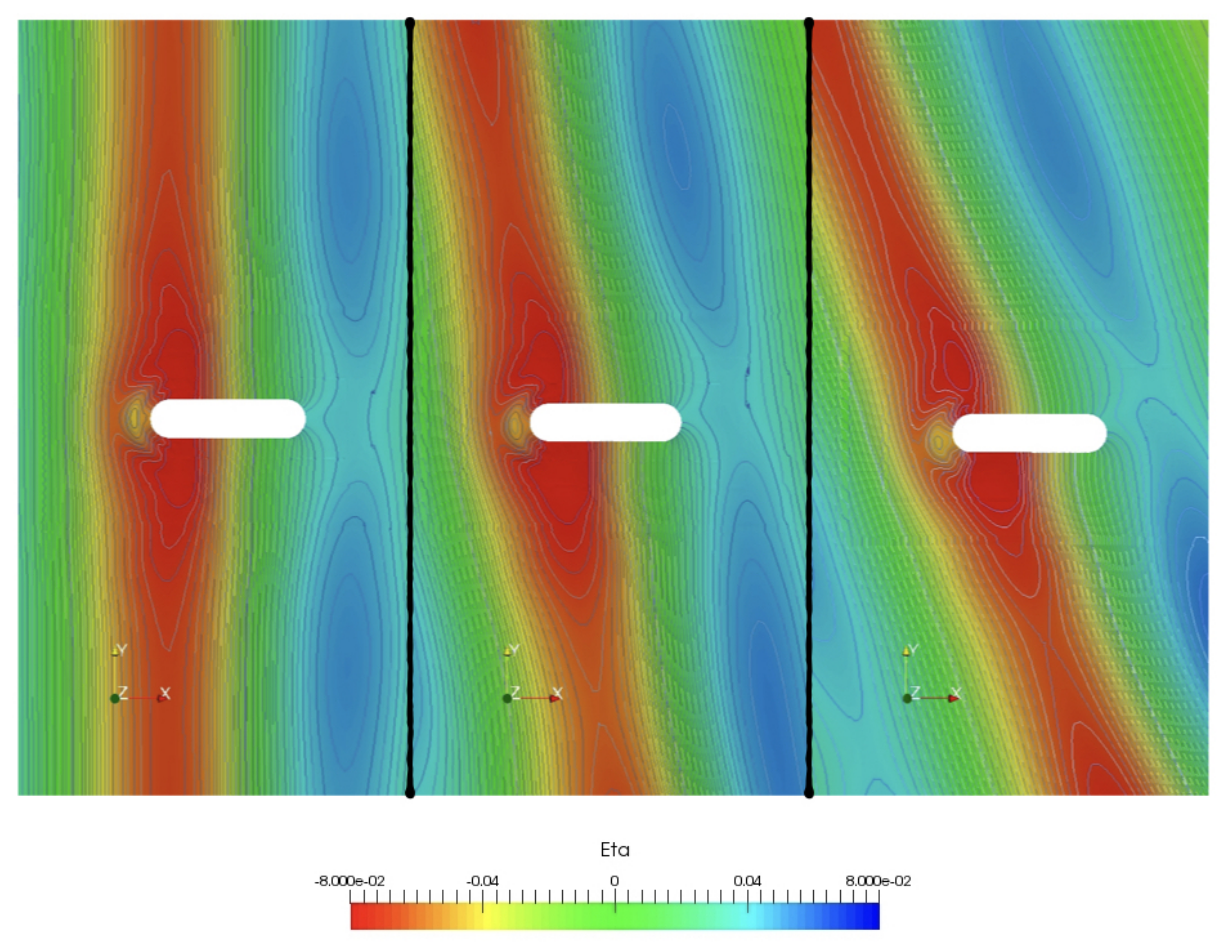

Figure 13: The surface elevation contour near the FPSO under the propagation angle $\beta=0^{\circ}$ (left), $\beta=10^{\circ}$ (middle) and $\beta=20^{\circ}$ (right). The unit shown in the figure legend for surface elevation Eta is meter.

shown. Due to geometric and flow symmetry, the transverse force component is zero for the case with $\beta=0^{\circ}$. However, with the increase of the incident angle to $\beta=20^{\circ}$, the transverse component rapidly grows to the same order of magnitude as the longitudinal force component. Meanwhile, the longitudinal force component remains nearly the same. This indicates that in the practical design, the angle effect should be taken into account, as the moment of inertia for the FPSO in the transverse direction is much lower than in the longitudinal direction.

\section{Summary and conclusions}

This paper presents a numerical study of the generation of oblique focused wave groups and their interaction with a fixed FPSO-shape body. The generation scheme is based on the snake principle, where the surface elevation and the velocity profiles are imposed on each virtual wave paddle. The wave propagation angle is controlled by the phase lag between the neighbouring wave paddles.

The focused wave groups are first generated in an empty wave basin without the presence of the FPSO. The surface elevations at a number of positions are compared with the available experimental data. It is found that the numerical results agree best with the experimental data when $\beta=0^{\circ}$, where the differences in surface elevation are up to $7 \%$. The discrepancy is likely to increase with the increase of the incident angle and the differences in the surface elevation reach up to $15 \%$ for $\beta=20^{\circ}$. Further analysis on the harmonic structure of each wave group shows that the first and second order harmonics agree with each other fairly well, but significant discrepancies occur for the third order harmonic, although its magnitude (less than $2 \% A$ ) is fairly small.

In the final part of the paper, we present the results from the simulation of oblique focused wave groups interaction with a fixed FPSO-shaped body, from which the similar trend can be observed, i.e. with the increase of the incident angle, the differences between the numerical model and the experimental data become larger and the numerical model tends to underestimate the angle effect. The integrated forces on the FPSO 

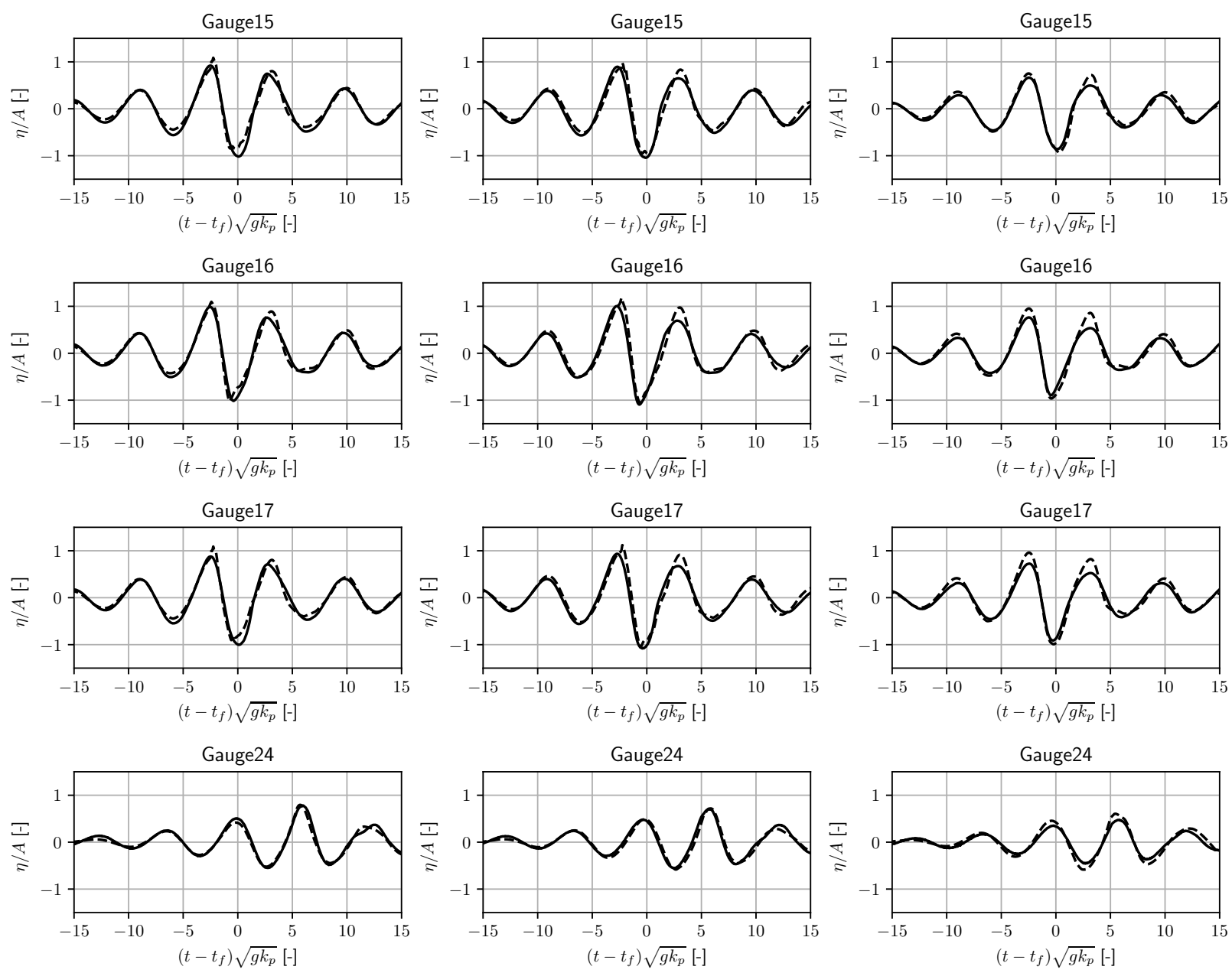

Figure 14: Comparison of the surface elevation at selected wave gauges between the numerical and experimental results for the case of oblique focused wave groups propagating obliquely in the wave tank with FPSO in place. Left column: $\beta=0^{\circ}$. Middle column: $\beta=10^{\circ}$. Right column: $\beta=20^{\circ}$. The location of the wave gauges is presented in Fig. 5 Solid line: experimental results. Dashed line: numerical results from OpenFOAM.

are also presented and a strong angle dependence of the transverse force component is observed, e.g. when the incident wave angle is increased to $20^{\circ}$, the transverse component is in the same order of magnitude as the longitudinal force component, while it is effectively zero when $\beta=0^{\circ}$.

The present work demonstrates the capability of the numerical wave basin for studying directional waves and their effects on dynamic loading on FPSOs. In the future, the developed methods will be extended for more complex cases of the generation of multi-directional irregular waves or focused wave groups, involving both frequency and direction spectrum, and applied to study their loading on and dynamic responses of offshore and ocean structures under extreme sea states.

\section{Acknowledgement}

The physical data presented here was generated as part of EPSRC funded research (EP/J012866/1) and is distributed via the CCP-WSI (EP/M022382/1) through the CCP-WSI project website (http://www.ccpwsi.ac.uk/). This research was supported by the Engineering and Physical Sciences Research Council (EPSRC), U.K. Project: A Zonal CFD Approach for Fully Nonlinear Simulations of Two Vessels in Launch and 

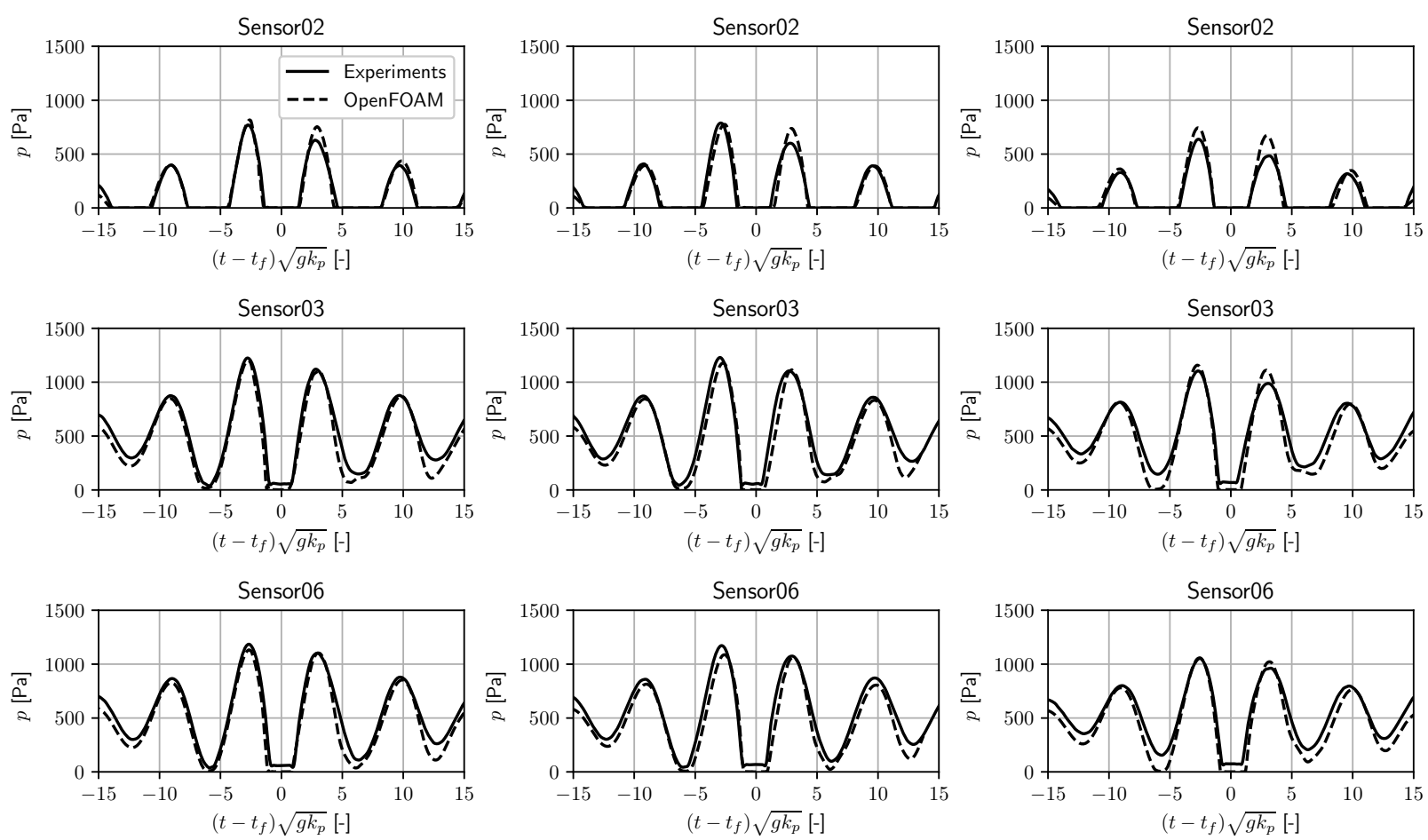

Figure 15: Comparison of the local pressure measured from the numerical model and the experiments. Left column: $\beta=0{ }^{\circ}$. Middle column: $\beta=10^{\circ}$. Right column: $\beta=20^{\circ}$. The location of the pressure sensor is presented in Fig. 6

Recovery Operations, under Grant No. EP/N008839/1. The authors would like to thank the anonymous reviewers for their useful comments and suggestions, which have certainly helped improve the paper. The support from a visiting foreign expert programme of Hohai University, China, under the project number T2018049, is also acknowledged by the second and the last authors.

\section{Appendix A: Three-dimensional wave-maker motion based on the snake principle}

In the laboratory, the oblique waves are generated via a number of segmented wave paddles, where the phase of each paddle is carefully adjusted based on the snake principle. This section briefly summarises the key points of the principle, and readers are referred to Dean and Dalrymple (1991) and Frigaard et al. (1993) for further details.

For a plane wave-maker which generates progressive waves normal to the wave-maker direction, the wave-maker motion can be obtained by solving the linear boundary value problem. This assumes that the flow is irrotational and inviscid. Therefore, the governing equations reduce to the Laplacian equation with certain boundary conditions. For linear wave-maker problems, the boundary conditions remain the same as other typical linear water wave problems at the seabed and the linearised free surface. However, at the wave-maker side, the boundary condition becomes

$$
F(x, z, t)=x-\frac{S(z)}{2} \sin \omega t=0
$$

By proper linearisation, this boundary condition is reduced to

$$
u(0, z, t)=\frac{S(z)}{2} \omega \cos \omega t
$$



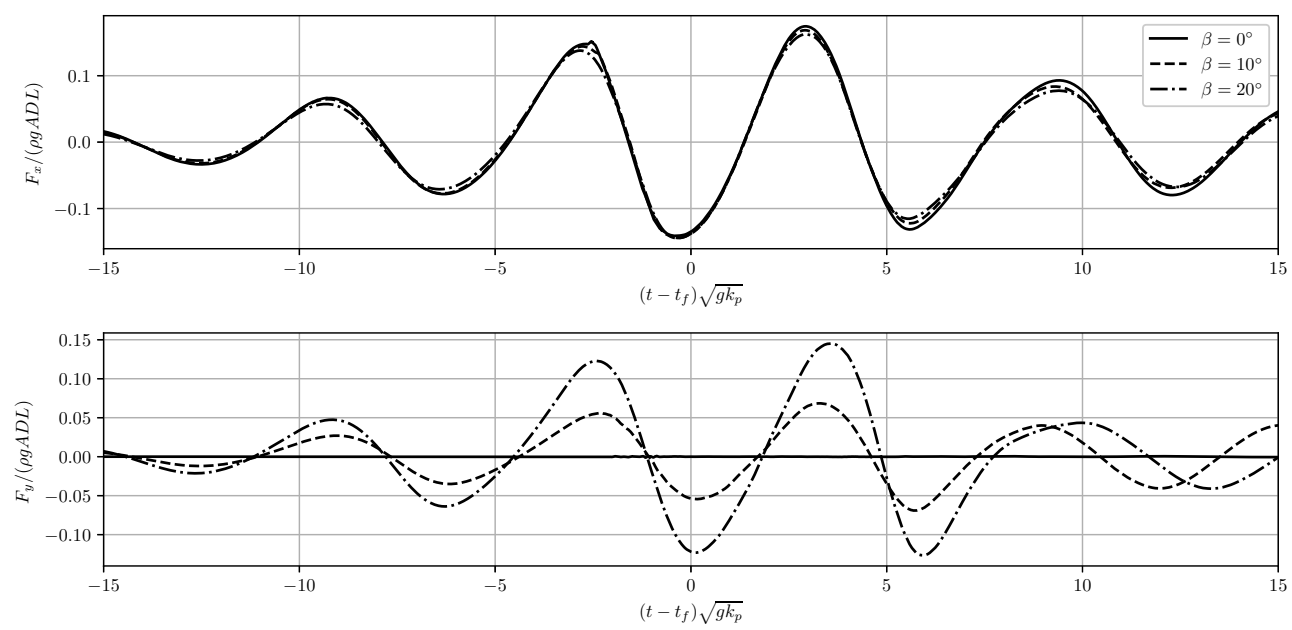

Figure 16: Numerical results of the longitudinal and transverse force components on the FPSO surface under three different incident wave angles.

Solution of this problem requires separation of variable, leading to the final velocity potential as shown below:

$$
\phi(x, z, t)=A \cosh k(h+z) \sin (k x-\omega t)+\sum_{n=1}^{\infty} C_{n} e^{-k_{s}(n) x} \cos \left[k_{s}(n)(h+z)\right] \cos \omega t
$$

where $A$ and $C$ are the parameters determined by the wave-maker motions. This velocity potential is comprised of two components, namely the progressive wave mode and the evanescent modes, where $k_{s}$ is the wave number for the evanescent wave modes. These evanescent modes decay soon within two to three water depth distance. Therefore, given a proper transfer function, i.e. $H / S$ where $H$ is the wave height and $S$ is the stroke of the wave-maker, the elevation far away from the wave-maker coincides with the linear progressive wave:

$$
\eta=\frac{H}{2} \cos (k x-\omega t)
$$

In 3D case, plane progressive waves propagating in different directions can be generated by using an articulated long wave-makers in a 3D wave basin. By assuming that the wave-maker is infinitely long and the length of the wave paddles is negligible, the following boundary condition can be applied:

$$
u(0, y, z, t)=U(z) \cos (k y \sin \beta-\omega t)
$$

By examining all the possible solutions, only one form involves a progressive wave mode which reads:

$$
\begin{aligned}
\phi= & A^{\prime} \cosh k(h+z) \sin (k x \cos \beta+k y \sin \beta-\omega t) \\
& +\sum_{n=1}^{\infty} C_{n} \cos \left[k_{s}(n)(h+z)\right] \exp \left[-\sqrt{k_{s}^{2}(n)+(k \sin \beta)^{2}} x\right] \cos (k y \sin \beta-\omega t)
\end{aligned}
$$

Note that $A^{\prime}$ is related to $A$ by $\cos ^{-1} \beta$. This corresponds to the surface elevation far away from the wave-maker:

$$
\eta=\frac{H^{\prime}}{2} \cos (k x \cos \beta+k y \sin \beta-\omega t)
$$

where $H^{\prime}=H / \cos \beta$. 

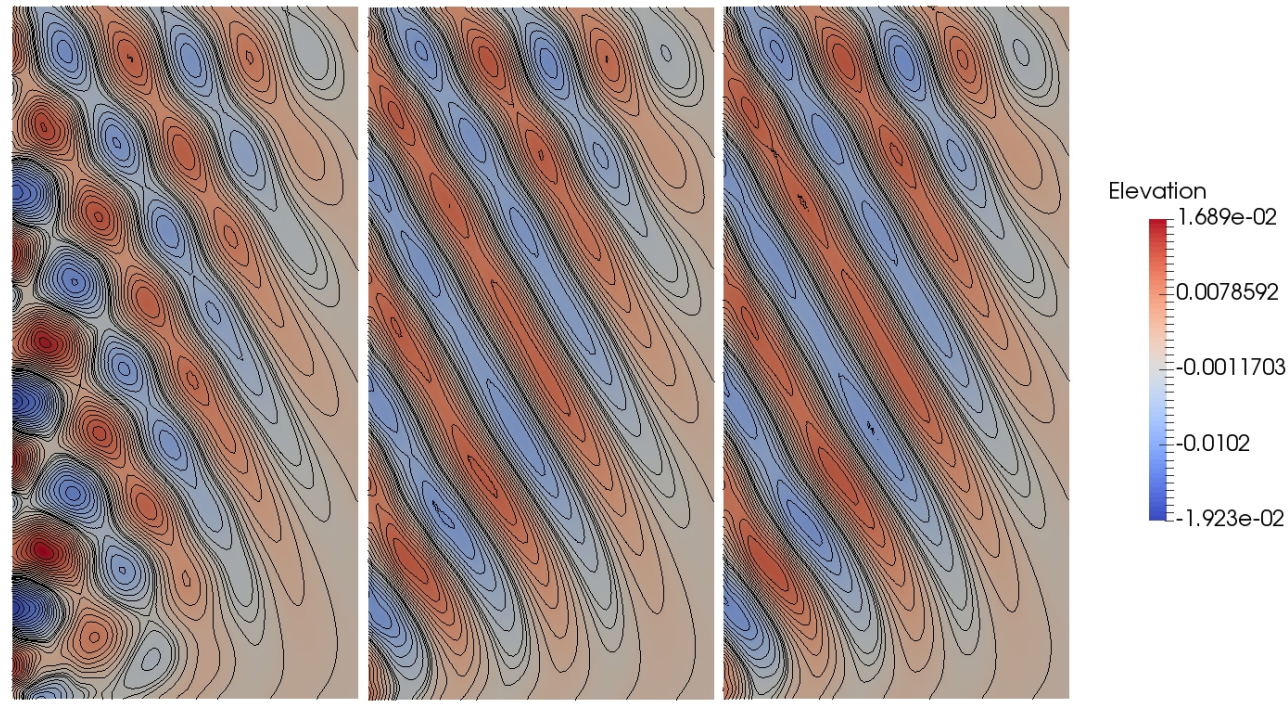

Figure 17: Free surface contour for directional linear regular wave propagating in $30^{\circ}$ with the target wave condition of $H=0.02$ $\mathrm{m}$ and $T=1 \mathrm{~s}$ with different wave paddles at the wave-maker side. The domain size is $5 \mathrm{~m}$ long and $10 \mathrm{~m}$ wide. The water depth $h$ is set to $0.543 \mathrm{~m}$. The paddle number at the wave-maker side is 10 (left), 20 (middle) and 30 (right).

\section{Appendix B: Sensitivity tests}

This section presents the sensitivity tests of several important parameters related to the numerical model, which includes the paddle number, mesh and time step convergence and the wave-maker length. Furthermore, we also examine the difference of the numerical solution between using reflective side walls and absorptive side walls.

\section{Paddle number}

The paddle number at the wave-maker side is important to both the numerical and experimental solutions, as insufficient paddle at the wave-maker side can lead to bumpy wave field, especially in the near field close to the wave-maker. Numerical experiments with three different paddle numbers, namely 10, 20 and 30 , are conducted. This corresponds to a paddle width of $0.67,0.33$ and $0.22 L$, where $L$ is the wave length, as the target regular wave condition is $H=0.02 \mathrm{~m}$ and $T=1 \mathrm{~s}$. The water depth is set to $0.543 \mathrm{~m}$.

Fig. 17 shows the surface elevation contour using different number of wave paddles. With a paddle width of $0.67 \mathrm{~L}$, the numerical wave tank can not produce a smooth elevation profile, especially in the area near the wave-maker, where standing waves are formed. This is due to the finite width effect of the wave paddle, as essentially the wave-maker motion is discontinuous, which significantly deviates from the assumption of the snake-principle. However, the wave field is becoming smoother with increasing paddle number at the wave-maker side. The difference between the cases using 20 and 30 wave paddles is nearly invisible, indicating that the solution is convergent. Hereby it is recommended that the width of the paddle should not exceed to $0.33 \mathrm{~L}$.

\section{Wave-maker length}

The wave-maker length is a critical parameter to the quality of the generated waves. Fig. 18 depicts the wave elevation contour with length of $2 \mathrm{~m}, 5 \mathrm{~m}, 10 \mathrm{~m}$ and $20 \mathrm{~m}$ on the wave-maker side, corresponding to $1.31,3.29,6.58$ and $13.16 \mathrm{~L}$. It is clearly seen that when the wave-maker side is reduced to $1.31 \mathrm{~L}$, the side wall has significant effects on the wave profiles. A curved wave front is generated instead of a front with straight line. With increasing wave-maker length, this wave front tends to form a straight line far away from the side walls, where the curvature eventually disappears. Actually with a wave-maker length of $6.58 \mathrm{~L}$, the generated oblique wave field is fine enough, which is recommended to use in the simulations. 


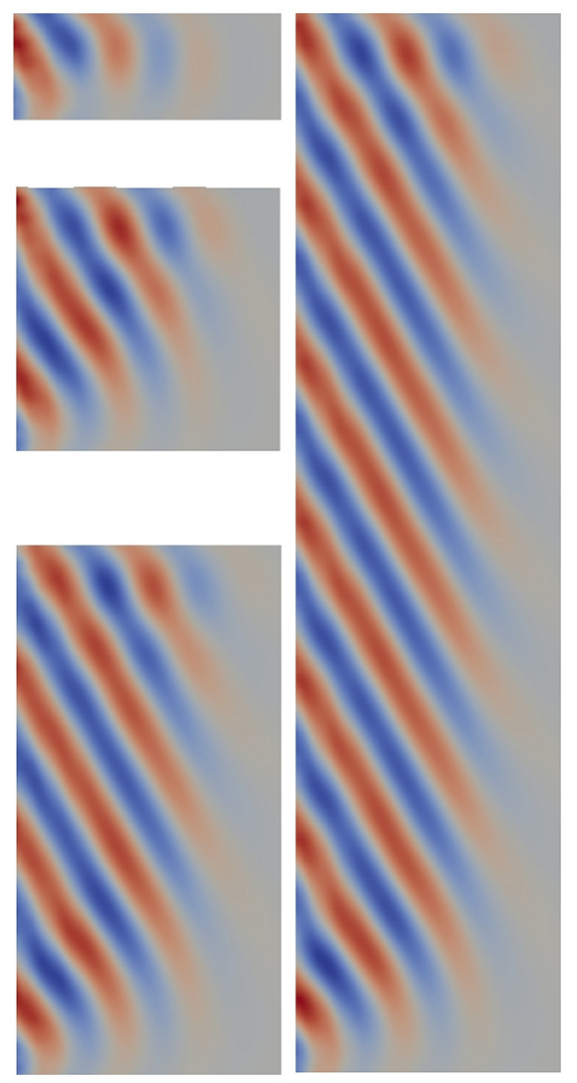

Figure 18: Free surface contour for directional linear regular wave propagating in $30^{\circ}$ with the target wave condition of $H=0.02$ $\mathrm{m}$ and $T=1 \mathrm{~s}$ with different wave-maker length. The water depth $h$ is set to $0.543 \mathrm{~m}$. The length of the domain is $5 \mathrm{~m}$, and the width of the domain is $2 \mathrm{~m}$ (left upper), $5 \mathrm{~m}$ (left middle), $10 \mathrm{~m}$ (left lower) and $20 \mathrm{~m}$ (right).

\section{Reflective side walls vs. absorptive side walls}

In the physical laboratory, solid walls were arranged at the side, which is reflective. However, in the numerical model, one can choose to use either reflective wall or absorptive wall (the active absorption boundary condition in IHFOAM) as the boundary condition. Hereby we test the difference of the numerical solutions using these two boundary conditions. In this case the propagation angle is $20^{\circ}$. Exactly the same boundary conditions and numerical parameters are used, except at the side walls.

The surface elevation at Gauge 11 is plotted in Fig. 19. The difference is visually negligible. This is reasonable as the central area which is of our interest is far away from the side wall. Furthermore, even if an active absorption boundary is applied, one should not expect that the $y$ component of the wave velocity is all absorbed. Therefore, the overall difference using these two boundary conditions is rather small.

\section{Computational mesh}

Sensitivity tests on the computational mesh is presented in this section. The tested case is for the focused wave with $\beta=0^{\circ}$ propagating in the empty tank. Totally four different mesh resolutions are used in the sensitivity analysis, where the ratio of the cell size is $1 / \sqrt{2}$ between the neighbouring resolutions, as shown in Table 3 Therefore, this test covers a refinement factor of 22.8 , as the ratio of mesh number between the finest and the coarsest mesh. For all the meshes, the cells are uniformly distributed in $x$ and $y$ directions. In the vertical $z$ direction, the cells are stretched to achieve a relatively fine mesh at the free surface area. The geometric similarity between the meshes are ensured.

Comparison of the surface elevation at the theoretical focal position (Gauge 11) is presented in Fig. 20 The numerical solutions are found to be unlikely converged even with the finest resolution. This is due to 


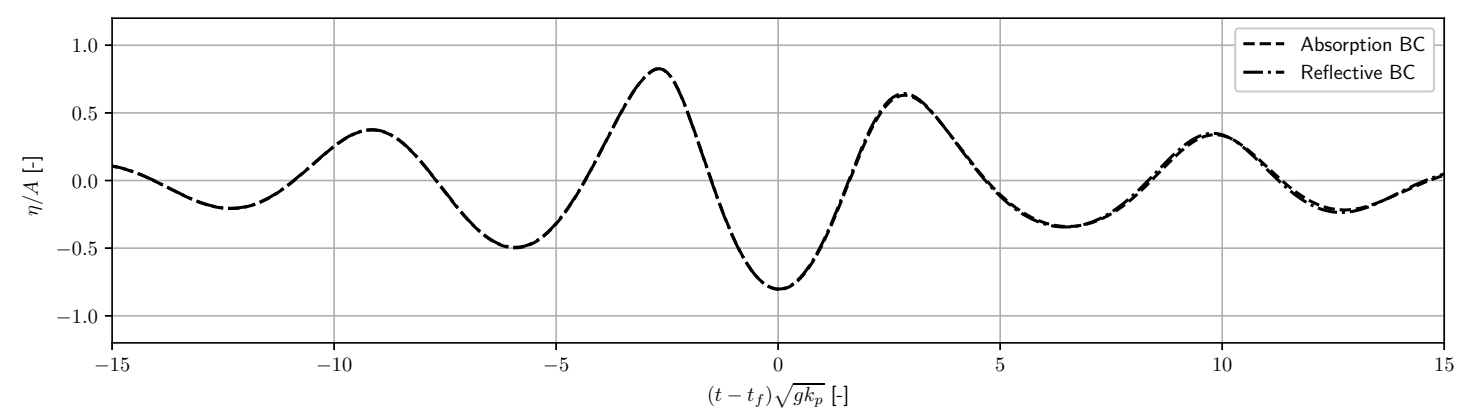

Figure 19: Comparison of the surface elevation at Gauge 11 between using reflective side walls and absorptive side walls.

Table 3: The mesh resolutions used in the convergence analysis for the numerical model. $\Delta x, \Delta y$ and $\Delta z$ are the grid size at the refined free surface area. Co is the maximum allowed Courant number. $\Delta t$ is the typical time step used in the simulation near the focal time. $\eta_{\min }$ is the surface elevation at the focal time.

\begin{tabular}{cccccccc}
\hline Grid ID & $L_{p} / \Delta x[-]$ & $L_{p} / \Delta y[-]$ & $A / \Delta z[-]$ & Mesh NO. [-] & Co. NO. [-] & $\Delta t[\mathrm{~s}]$ & $\eta_{\min } / A[-]$ \\
1 & 14.60 & 13.27 & 7.41 & 0.80 million & 0.25 & $\sim 0.0030$ & 0.708 \\
2 & 20.85 & 18.02 & 10.22 & 2.27 milliom & 0.25 & $\sim 0.0025$ & 0.786 \\
3 & 29.20 & 25.61 & 14.35 & 6.48 million & 0.25 & $\sim 0.0018$ & 0.866 \\
4 & 41.24 & 36.50 & 20.32 & 18.26 milliom & 0.25 & $\sim 0.0015$ & 0.935 \\
\hline
\end{tabular}

the reason that the domain in the numerical model is fairly large, comparing with normal Navier-Stokes solver based numerical models. Even with 18 million cells, the resolution can be somehow only defined as moderately fine, especially in $x$ and $y$ direction. This may lead to both dispersion and diffusion errors. The dispersion error is due to the difference of the resolved celerities when using different resolutions. This can be seen in Fig. 20 that there is a clear discrepancy on the crests close to the neighbouring trough, in the perspective of occurrence time. Meanwhile, the difference in the wave height can be owed to the diffusion error. which are shown in both the focal trough and the neighbouring crests.

Actually focused wave group is rather complex and not straightforward to model, as they contain many different components, which are induced by the wave-maker motion and the wave-wave interaction during the evolution. While the mesh can resolve most of the energetic components near the peak frequency, the components at the tail of the spectrum are difficult to resolve. Considering that a JONSWAP spectrum has a relatively long tail, which probably makes the convergence more difficult.

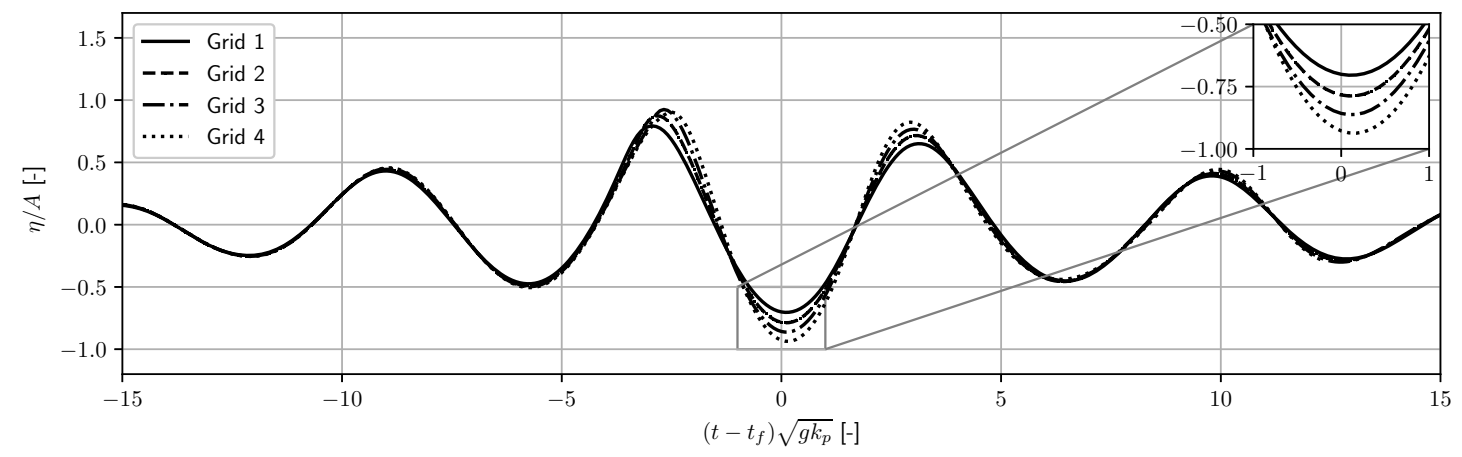

Figure 20: The surface elevation at Gauge 11 using four different grid resolutions. The resolutions are given in Table 3 and the position of Gauge 11 is given in Fig. 4 
Table 4: The mesh resolutions used in the convergence analysis for the numerical model. $\Delta x, \Delta y$ and $\Delta z$ are the grid size at the refined free surface area. Co is the maximum allowed Courant number. $\Delta t$ is the typical time step used in the simulation near the focal time. $\eta_{\min }$ is the surface elevation at the focal time.

\begin{tabular}{cccccccc}
\hline Case ID & $L_{p} / \Delta x[-]$ & $L_{p} / \Delta y[-]$ & $A / \Delta z[-]$ & Mesh NO. [-] & Co. NO. [-] & $\Delta t[\mathrm{~s}]$ & $\eta_{\min } / A[-]$ \\
1 & 29.20 & 25.61 & 14.35 & 6.48 million & 0.45 & $\sim 0.0010$ & 0.822 \\
2 & 29.20 & 25.61 & 14.35 & 6.48 milliom & 0.35 & $\sim 0.0030$ & 0.863 \\
3 & 29.20 & 25.61 & 14.35 & 6.48 million & 0.25 & $\sim 0.0018$ & 0.866 \\
4 & 29.20 & 25.61 & 14.35 & 6.48 milliom & 0.15 & $\sim 0.0007$ & 0.884 \\
\hline
\end{tabular}

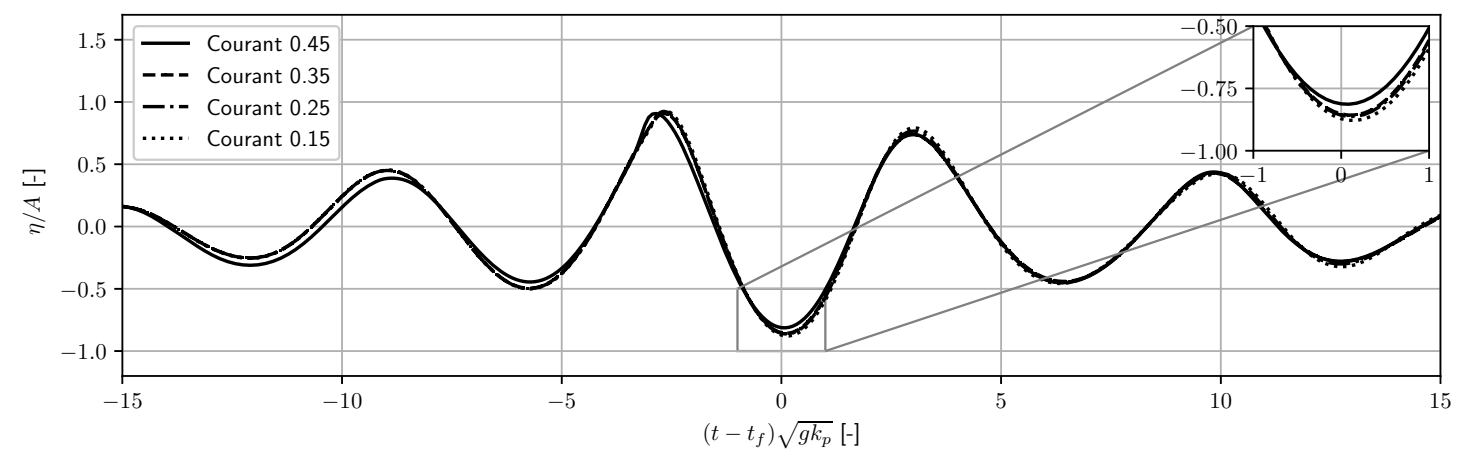

Figure 21: The surface elevation at Gauge 11 using four different Courant numbers.

\section{Time step}

Convergence tests are also carried out for the time step used in the simulations. As adjustable time step is applied based on the limitation of Courant number, here the characteristic time step is also represented by the maximum Courant number that is allowed during the computation. Four different Courant numbers are used in the convergence test, namely $0.45,0.35,0.25$ and 0.15 , while the same mesh resolution is applied, i.e. Grid 3. The relevant parameters for this convergence test is given in Table 4 and the results are shown in Fig. 21. Unlike mesh resolution, the numerical results are well converged for different Courant number limits when it is below 0.35. The difference of the surface elevation at the focal time between Case 2 and Case 4 is $2 \%$, indicating that the results are independent of the time step that is applied in the simulations. Moreover, it is noted that the time step used in Case 1 is significantly smaller than in Case 2. This somehow indicates that certain stability issues arise, resulting in spurious large velocities which limit the time step to unreasonably small.

\section{References}

Bai W, Eatock Taylor R. Higher-order boundary element simulation of fully nonlinear wave radiation by oscillating vertical cylinders. Applied Ocean Research 2006;28(4):247-65.

Bai W, Eatock Taylor R. Numerical simulation of fully nonlinear regular and focused wave diffraction around a vertical cylinder using domain decomposition. Applied Ocean Research 2007;29(1-2):55-71.

Berberović E, van Hinsberg N, Jakirlić S, Roisman I, Tropea C. Drop impact onto a liquid layer of finite thickness: Dynamics of the cavity evolution. Physical Review E 2009;79(3):036306. doi 10.1103/PhysRevE.79.036306

Cao HJ, Wan DC. Development of multidirectional nonlinear numerical wave tank by naoe-foam-sjtu solver. International Journal of Ocean System Engineering 2014;4(1):49-56.

Chabchoub A, Hoffmann N, Akhmediev N. Rogue wave observation in a water wave tank. Physical Review Letters 2011;106(20):204502.

Chen L, Zang J, Taylor PH, Sun L, Morgan G, Grice J, Orszaghova J, Ruiz MT. An experimental decomposition of nonlinear forces on a surface-piercing column: Stokes-type expansions of the force harmonics. Journal of Fluid Mechanics 2018;848:4277.

Chen X. Development of three dimensional numerical wave basin for simulation of extreme events in ocean. Ph.d. thesis; National University of Singapore; 2017.

Christou M, Ewans K. Field measurements of rogue water waves. Journal of Physical Oceanography 2014;44(9):2317-35. 
Dao MH, Chew LW, Zhang Y. Modelling physical wave tank with flap paddle and porous beach in OpenFOAM. Ocean Engineering 2018;154(February):204-15. doi 10.1016/j.oceaneng.2018.02.024

Dean RG, Dalrymple RA. Water wave mechanics for engineers and scientists. volume 2. World Scientific Publishing Company, 1991.

Ducrozet G, Bonnefoy F, Le Touzé D, Ferrant P. Implementation and validation of nonlinear wavemaker models in a HOS numerical wave tank. International Journal of Offshore and Polar Engineering 2006;16(3):161-7.

Dysthe K, Krogstad HE, Müller P. Oceanic Rogue Waves. Annual Review of Fluid Mechanics 2008;40(1):287-310. doi 10. 1146/annurev.fluid.40.111406.102203

Fitzgerald CJ, Taylor PH, Eatock Taylor R, Grice J, Zang J. Phase manipulation and the harmonic components of ringing forces on a surface-piercing column. Proceedings of the Royal Society A: Mathematical, Physical and Engineering Sciences 2014;470(2168):20130847-. doi 10.1098/rspa.2013.0847

Frigaard P, Hogedal M, Christensen M. Wave Generation Theory. Denmark: Hydraulics \& Coastal Engineering Laboratory, Department of Civil Engineering, Aalborg University, 1993.

Ha T, Lin P, Cho YS. Generation of 3D regular and irregular waves using Navier-Stokes equations model with an internal wave maker. Coastal Engineering 2013;76:55-67. doi 10.1016/j.coastaleng.2013.01.013

Halliday J, Dorrell D. Review of wave energy resource and wave generator developments in the uk and the rest of the world. In: Proceedings of the 4th IASTED International Conference on Power and Energy Systems. 2004. p. 76-83.

Hasselmann K, Barnett T, Bouws E, Carlson H, Cartwright D, Enke K, Ewing J, Gienapp H, Hasselmann D, Kruseman P, et al. Measurements of wind-wave growth and swell decay during the joint north sea wave project (jonswap). Ergänzungsheft 8-12 1973;.

Higuera P, Lara JL, Losada IJ. Realistic wave generation and active wave absorption for Navier-Stokes models. Application to OpenFOAMR. Coastal Engineering 2013;71:102-18. doi 10.1016/j.coastaleng.2012.07.002

Higuera P, Losada IJ, Lara JL. Three-dimensional numerical wave generation with moving boundaries. Coastal Engineering 2015;101:35-47. doi $10.1016 / \mathrm{j}$.coastaleng.2015.04.003

Hirt C, Nichols B. Volume of fluid (VOF) method for the dynamics of free boundaries. Journal of Computational Physics 1981;39(1):201-25. doi 10.1016/0021-9991(81)90145-5

Hu ZZ, Greaves D, Raby A. Numerical wave tank study of extreme waves and wave-structure interaction using openfoam $\mathbb{R}$. Ocean Engineering 2016;126:329-42.

Issa RI. Solution of the implicitly discretised fluid flow equations by operator-splitting. Journal of Computational Physics 1986;62(1):40-65. doi 10.1016/0021-9991(86)90099-9

Ji X, Liu S, Bingham HB, Li J. Multi-directional random wave interaction with an array of cylinders. Ocean Engineering 2015a;110:62-77. doi 10.1016/j.oceaneng.2015.09.039

Ji X, Liu S, Li J, Jia W. Experimental investigation of the interaction of multidirectional irregular waves with a large cylinder. Ocean Engineering 2015b;93:64-87. doi 10.1016/j.oceaneng.2014.10.004

Karjanto N, Van Groesen E. Qualitative comparisons of experimental results on deterministic freak wave generation based on modulational instability. Journal of hydro-environment research 2010;3(4):186-92.

Kim M, Niedzwecki J, Roesset J, Park J, Hong S, Tavassoli A. Fully nonlinear multidirectional waves by a 3-d viscous numerical wave tank. Journal of Offshore Mechanics and Arctic Engineering 2001;123(3):124-33.

Li J, Chen G, Yang J, Peng T. Laboratory research on effective test area of short-crested waves generated by two-sided segmented wavemakers. China Ocean Engineering 2014a;28(2):181-92. doi 10.1007/s13344-014-0014-7

Li J, Wang Z, Liu S. Experimental study of interactions between multi-directional focused wave and vertical circular cylinder, Part I: Wave run-up. Coastal Engineering 2012;64:151-60. URL: http://dx.doi.org/10.1016/j.coastaleng.2012.02. 003https://linkinghub.elsevier.com/retrieve/pii/S0378383912000270 doi 10.1016/j.coastaleng.2012.02.003

Li J, Wang Z, Liu S. Experimental study of interactions between multi-directional focused wave and vertical circular cylinder, part II: Wave force. Coastal Engineering 2014b;83:233-42. URL: http://dx.doi.org/10.1016/j.coastaleng.2013.06. 004https://linkinghub.elsevier.com/retrieve/pii/S0378383913001129 doi 10.1016/j.coastaleng.2013.06.004

Li Q, Yan S, Wang J, Ma Q, Xie Z, Sriram V, et al. Numerical simulation of focusing wave interaction with fpso-like structure using fnpt-ns solver. In: The 28th International Ocean and Polar Engineering Conference. International Society of Offshore and Polar Engineers; 2018.

Li Y, Liu SX, Yu YX, Lai GZ. Numerical modelling of multi-directional irregular waves through breakwaters. Applied Mathematical Modelling 2000;24(8):551 -74. doi https://doi.org/10.1016/S0307-904X(00)00003-2

Mai T, Greaves D, Raby A, Taylor PH. Physical modelling of wave scattering around fixed FPSO-shaped bodies. Applied Ocean Research 2016;61:115-29. doi 10.1016/j.apor.2016.10.007

Martínez-Ferrer PJ, Qian L, Ma Z, Causon DM, Mingham CG. Improved numerical wave generation for modelling ocean and coastal engineering problems. Ocean Engineering 2018;152(September 2017):257-72. URL: https://doi.org/10.1016/j. oceaneng.2018.01.052 doi $10.1016 / \mathrm{j}$.oceaneng.2018.01.052.

Newman JN. Analysis of wave generators and absorbers in basins. Applied Ocean Research 2010;32(1):71-82. doi 10.1016/j . apor.2010.04.004

Ning DZ, Zang J, Liu SX, Eatock Taylor R, Teng B, Taylor PH. Free-surface evolution and wave kinematics for nonlinear uni-directional focused wave groups. Ocean Engineering 2009;36(15-16):1226-43. URL: http://dx.doi.org/10.1016/j . oceaneng.2009.07.011 doi 10.1016/j.oceaneng.2009.07.011.

Ojieh N, Barltrop N, Xu L. Rans investigation of the kinematics of an alternative extreme wave. Ocean Engineering 2009;36(1718):1415-24.

O'Dea JF, Newman JN. Numerical studies of directional wavemaker performance. In: 28th American Towing Tank Conference, Ann Arbor, Michigan. 2007. . 
Peregrine D. Water waves, nonlinear schrödinger equations and their solutions. The ANZIAM Journal 1983;25(1):16-43. Rhie C, Chow W. A NUMERICAL STUDY OF THE TURBULENT FLOW PAST AN ISOLATED. Technical Report; 1982. Swan C, Sheikh R. The interaction between steep waves and a surface-piercing column. Philosophical Transactions of the Royal Society A: Mathematical, Physical and Engineering Sciences 2015;373(2033):20140114.

Tromans PS, Anaturk AR, Hagemeijer P. A new model for the kinematics of large ocean waves-application as a design wave. In: The First International Offshore and Polar Engineering Conference. International Society of Offshore and Polar Engineers; 1991. .

Vyzikas T, Stagonas D, Buldakov E, Greaves D. The evolution of free and bound waves during dispersive focusing in a numerical and physical flume. Coastal Engineering 2018;132(February 2017):95-109. doi 10.1016/j.coastaleng.2017.11.003.

Williams AN, Crull WW. Simulation of directional waves in a numerical basin by a desingularized integral equation approach. Ocean Engineering 2000;27(6):603-24. doi 10.1016/S0029-8018(99)00016-5. 